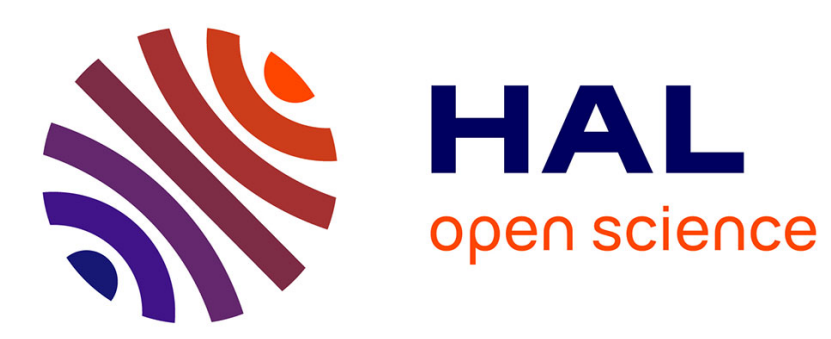

\title{
Polydisperse polymer networks : elasticity, orientational properties, and small angle neutron scattering
}

P.G. Higgs, R.C. Ball

\section{To cite this version:}

P.G. Higgs, R.C. Ball. Polydisperse polymer networks: elasticity, orientational properties, and small angle neutron scattering. Journal de Physique, 1988, 49 (10), pp.1785-1811. 10.1051/jphys:0198800490100178500 . jpa-00210860

\section{HAL Id: jpa-00210860 https://hal.science/jpa-00210860}

Submitted on 1 Jan 1988

HAL is a multi-disciplinary open access archive for the deposit and dissemination of scientific research documents, whether they are published or not. The documents may come from teaching and research institutions in France or abroad, or from public or private research centers.
L'archive ouverte pluridisciplinaire HAL, est destinée au dépôt et à la diffusion de documents scientifiques de niveau recherche, publiés ou non, émanant des établissements d'enseignement et de recherche français ou étrangers, des laboratoires publics ou privés. 
Classification

Physics Abstracts

$61.40 \mathrm{~K}-61.12-78.20$

\title{
Polydisperse polymer networks : elasticity, orientational properties, and small angle neutron scattering
}

\author{
P. G. Higgs and R. C. Ball \\ Cavendish Laboratory, Madingley Road, Cambridge, CB3 0HE, G.B.
}

(Reçu le 11 avril 1988, accepté sous forme définitive le 20 juin 1988)

\begin{abstract}
Résumé. - Les propriétés des réseaux de polymères dépendent de la distribution $f(S)$ des longueurs de chaines entre jonctions. Nous développons un formalisme qui décrit les réseaux fantômes en permettant d'inclure explicitement une distribution $f(S)$ arbitraire. Dans le cas de distributions bimodes, nous étudions l'orientation des chaînes dans un réseau sous contrainte en calculant séparément pour les chaînes longues et courtes les distributions $P_{2}(\cos \chi)$ et $P_{4}(\cos \chi)$. Les réponses des deux espèces sont franchement différentes, quoique la biréfringence globale du réseau ne dépende que de la longueur de chaîne moyenne. De même, bien que le taux d'extension des chaînes d'un réseau sous contrainte soit très différent pour les deux expèces, le module du réseau reste formellement indépendant de $f(S)$. Nous étudions la diffusion de rayonnement par une chaîne longue marquée attachée au réseau en un grand nombre de points situés au hasard, et nous traitons explicitement la distribution de Poisson des longueurs entre jonctions. On calcule exactement la courbe de diffusion dans le cas simplifié où la déformation du système des points de jonction est affine ; pour le cas plus complexe où ces jonctions peuvent fluctuer librement, on peut trouver une solution approchée. Le premier de ces modèles (polydisperse, affin au niveau des jonctions) donne un meilleur accord avec les résultats expérimentaux que les théories existantes, aussi bien en ce qui concerne la forme de la courbe de Kratky que pour les contours iso-intensité. Cependant il subsiste un certain nombre de données qui ne peuvent être reproduites par aucun des modèles considérés. On montre par le calcul des courbes limites que ces différences ne peuvent être prises en compte que par un traitement qui va au-delà de l'idée du réseau fantôme.
\end{abstract}

Abstract. - The properties of polymer networks may be expected to depend upon the distribution, $f(S)$, of chain lengths between crosslinks. We develop a formalism for treating phantom networks which allows the explicit inclusion of an arbitrary $f(S)$ distribution. In bimodal networks we investigate chain orientation in the strained network by calculating $\left\langle P_{2}(\cos \chi)\right\rangle$ and $\left\langle P_{4}(\cos \chi)\right\rangle$ for the long and the short chains separately. The responses of the two species differ markedly from each other, although it is found that the overall network birefringence is dependent only on the mean chain length. Similarly, although the typical degree of extension of the chains upon straining the network differs widely between the species, the network modulus is formally independent of $f(S)$. We investigate scattering from a long labelled chain crosslinked in a network at many randomly-positioned points, and explicitly account for the Poisson distribution of lengths between crosslinks. The scattering function is calculated exactly if the simplification of affine deformation of the junction points is made, and if the junctions are allowed to fluctuate freely, then an approximate solution is possible. The former model (Polydisperse Junction Affine model) agrees much better with the experimental data than the existing theories, both as regards the shape of the Kratky plot and the iso-intensity contours. There remain, however, features of the data which cannot be reproduced by any of the models considered. It is shown by the calculation of limit curves that these differences can only be accounted for by a treatment which goes beyond the idea of phantom chains.

\section{Introduction.}

Theories of polymer networks often use the concept of the phantom network, in which the chains are assumed to interact only at certain permanent junction points (or crosslinks) [1-4]. It is possible to determine the properties of this simplified system in a way which would be extremely difficult if the full interactions, including topological entanglements etc., were taken into account.

A further approximation is usually made, namely that the chains comprising the network all have the 
same length. This is very clearly untrue for experimental systems (although progress has been made in producing monodisperse « model » networks [5]). It is assumed that experimentally measurable quantities, which involve an average over many chains, will not depend strongly on the variation in chain lengths which occurs, and that therefore it is valid to introduce a mean length at the beginning of the calculation. It is the object of this paper to investigate to what extent this monodisperse approximation is justified.

An alternative to the monodisperse approximation is provided by the replica method, introduced by Deam and Edwards [6]. Although this is a powerful general basis for calculation, the constraint of remaining analytically tractable forces other approximations. In particular the localizing effect on a chain of its crosslinks has been represented by a harmonic potential well. We will proceed using the phantom network since this will allow a clear comparison to be made between polydisperse and monodisperse theories without the influence of complicating factors such as entanglements, chain stiffness etc. We do not use the replica method explicitly but draw on some of its results.

\section{The phantom network.}

The phantom network has been discussed by many authors, the most comprehensive treatments being by Flory [1], and James and Guth [2-4]. We will here list the basic assumptions of the theory and discuss under what conditions we may expect them to be applicable :

(1) The network consists of subchains which are freely flexible and which have an end-to-end vector $\mathbf{r}$ described by the Gaussian probability distribution $p(\mathbf{r})$. (The word subchains is used since in general the network will be formed by irradiating or adding crosslinking agent to a melt of long chains, so that there will be more than one crosslink on any chain.) ;

(2) The subchains interact only at their end points ;

(3) The distribution $p(\mathbf{r})$ of end-to-end vectors for a subchain is the same in the unstrained network as it was in the uncrosslinked system under the same conditions of temperature, solution concentration etc. ;

(4) In this paper we assume that the arc lengths between crosslinks are governed by a probability distribution $f(S)$ which we may specify, that the lengths of neighbouring subchains are independent of each other, and that all crosslinks have the same functionality $\phi$.

Assumption 3 is discussed by Flory [1]. To see its validity we must consider the way the network was formed. We visualize an uncrosslinked melt just prior to crosslinking in which there are many points of contact, or near contact, between chains. The crosslinking process is seen as an instantaneous freezing-in of certain of these contact points chosen at random to become permanent junctions. The process introduces no stress into the system : it remains in equilibrium. The set of equilibrium configurations of the network is a subset of the configurations of the melt, and the subchain vector distribution remains unbiased by the crosslinking.

A further consequence of the above argument is that since there are many potential crosslink sites the chance of a chain linking with itself is very small. Also the chance of a subchain forming part of a closed loop containing only a small number of other subchains is very small. This is an important point in the analysis following in section 2 . We note that these arguments will not apply to all possible conditions under which networks form. For instance if the crosslinking occurs in dilute solution then a chain is much more likely to link to itself than to other molecules. There will be many small loops, and we would not expect the phantom network theory to apply so well.

The simplest form of the theory assumes that the junction points are fixed, and that they deform affinely, i.e. in direct proportion to the macroscopic strain. We refer to this as the Junction Affine network. James and Guth [2-4] modified the theory to allow junction fluctuations, and we refer to this case as the Phantom network.

Consider a subchain which can be represented as a random walk of $N$ steps of length $\ell$. Its end-to-end vector distribution is :

$$
p(\mathbf{r})=\frac{1}{(2 \pi S)^{3 / 2}} \exp \left(-\frac{\mathbf{r}^{2}}{2 S}\right)
$$

where

$$
S=N \ell^{2} / 3=\left\langle r^{2}\right\rangle / 3 .
$$

This equation defines $S$ to be the mean square value of any one component of the end-to-end vector. Since $S$ is proportional to the arc length of the subchain $(L=N \ell)$ we will from now on refer to « subchains of length $S$ ». This is the natural way of defining subchain lengths, since $\left\langle r^{2}\right\rangle$ can be measured experimentally whereas $N$ and $\ell$ are less easily obtainable.

For the subchain linking junctions $\mu$ and $\nu$ we may write :

$$
\mathbf{r}_{\mu \nu}=\mathbf{R}_{\mu \nu}+\rho_{\mu \nu}
$$

where $\mathbf{R}_{\mu \nu}$ is the mean end-to-end vector for the chain and $\rho_{\mu \nu}$ is a fluctuation about the mean. $\mathbf{R}_{\mu \nu}$ is not in general zero - the network does not collapse to a point. For each subchain the value of $\mathbf{R}_{\mu \nu}$ remains fixed, and the distribution of $\mathbf{r}_{\mu \nu}$ is 
therefore not equal to $p(\mathbf{r})$. The distribution $p(\mathbf{r})$ applies only when we consider the whole ensemble of subchains, with their ensemble of values for $\mathbf{R}_{\mu \nu}$.

James and Guth considered the problem of the introduction of the non zero mean vectors by using certain «fixed junctions » which determine the boundaries of the network. The mean vectors $\left\{\mathbf{R}_{\mu \nu}\right\}$ are then determined by the balancing of forces at each junction point. They showed that because of the properties of the Gaussian distributions for $\left\{\mathbf{r}_{\mu \nu}\right\}$ the fluctuations $\left\{\rho_{\mu \nu}\right\}$ also have Gaussian distributions and that the variances of these distributions are independent of the values of the mean vectors, and hence independent of the initial arbitrary choice of fixed junction positions. Flory [1] and Eichinger [7] have demonstrated that for the monodisperse network:

$$
\left\langle\rho^{2}\right\rangle=2 / \phi\left\langle r^{2}\right\rangle_{0}
$$

The subscript zero indicates the reference state in which the network was formed. We may define $S^{\text {eff }}$ to be the length of a free subchain which would have the same fluctuations as does the real subchain $S$ when it is constrained by the network.

$S^{\mathrm{eff}}=\left\langle\rho^{2}\right\rangle / 3$

$=2 / \phi S$ for a monodisperse network .

For a polydisperse network $\left\langle\rho^{2}\right\rangle$ will be different for each subchain, but it has been shown by Graessley [8] that in the absence of small loops (i.e. when the local topology is tree-like) the corresponding result :

$$
\left\langle S^{\mathrm{eff}} / S\right\rangle=2 / \phi
$$

applies for all subchain length distributions. We feel this is a key result whose implications have not been fully appreciated. We present an independent proof of it in appendix $\mathrm{A}$ in the language of the resistor network analogy to be introduced below.

It may be stated (refering to the derivations of [8] and [1]) that the free energy of the strained network relative to the unstrained state is

$$
F=\frac{1}{2} g N_{\mathrm{c}} k T\left(\sum_{i} \lambda_{i}^{2}-3\right)
$$

where $N_{\mathrm{c}}$ is the number of subchains per unit volume and the $\lambda_{i}$ are the extension ratios along the principal axes. The front factor $g$ is given by:

$$
g \equiv 1-\left\langle\frac{\rho^{2}}{r^{2}}\right\rangle \equiv 1-\left\langle\frac{S^{\mathrm{eff}}}{S}\right\rangle
$$

If the network may be approximated by a tree-like structure then, following Graessley [8], $g=1-2 / \phi$. This means that both the modulus and JOURNAL DE PHYSIQUE. - T. 49, N 10, OCTOBRE 1988 the shape of the stress-strain curve depend only on $N_{\mathrm{c}}$ and $\phi$ and are independent of the distribution $f(S)$ of subchain lengths. The shear modulus $G$ may be obtained from (1.7),

$$
G=N_{\mathrm{c}} k T(1-2 / \phi)
$$

and if we make the assumption of incompressibility then the Young's modulus is $E=3 G$. That the modulus should be independent of $f(S)$ is in clear disagreement with experiments on bimodal networks by Mark et al. [5, 14-17]. The experiments are discussed further in section 4 , but we note here that the experimental samples contain very short chains with very limited extensibility. The Gaussian theory would therefore not be expected to apply.

In deriving (1.7) the so-called additivity assumption is made, namely that the strain-dependent term in the free energy may be separated from all other contributions, such as polymer-polymer and polymer-solvent interactions. This is true only for deformations which preserve the volume of the sample, and providing the temperature and nature of the solvent are not changed from the conditions occurring during crosslinking. The effect of polymersolvent interactions and the validity of the additivity assumption are discussed by Ball and Edwards [9].

We note that the Junction Affine network corresponds to the $\phi=\infty$ limit of the phantom network, where $\rho^{2}=0$ for all subchains and $g=1$. The topology of the junction affine network is irrelevant since the chains are not affected by their neighbours. The role of topology in the phantom network is important. For example the regular tetrahedral lattice $(\phi=4)$ with all the chains the same length may be shown to have $g=1 / 2 \quad(=1-2 / \phi)$, whereas a regular cubic lattice $(\phi=6)$ with all chains the same length has $g=1 / 3 \quad(\neq 1-2 / \phi)$. Regular lattices maximize the number of small loops and are very unrealistic configurations. As discussed above, the conditions of formation are likely to prevent the formation of large numbers of small loops, and to make the tree approximation a good one.

The rest of this paper is concerned with calculating quantities which depend on the distribution of values of $S^{\text {eff }}$ rather than merely the average occurring in (1.8). We retain the tree approximation, noting that the whole of what follows is dependent on it. A solution for the opposite extreme of the regular lattice has yet to be found when the chains are polydisperse.

\section{Fluctuations in the polydisperse network.}

2. 1 THE RESISTOR NETWORK ANALOGY. - The variables $\left\{x_{\mu}, y_{\mu}, z_{\mu}\right\}$ representing the coordinates of the junctions have a joint probability distribution 
which is the quadratic form

$$
\begin{aligned}
& P\left(\left\{x_{\mu}, y_{\mu}, z_{\mu}\right\}\right) \propto \prod_{\mu \neq \nu} \exp \left\{-\frac{1}{2 S_{\mu \nu}} \times\right. \\
& \left.\times\left(\left(x_{\mu}-x_{\nu}\right)^{2}+\left(y_{\mu}-y_{\nu}\right)^{2}+\left(z_{\mu}-z_{\nu}\right)^{2}\right)\right\} \\
& \text { i.e. } P\left(\left\{x_{\mu}\right\}\right) \propto \exp \left(-\frac{1}{2} x_{\mu} A_{\mu \nu} x_{\nu}\right)
\end{aligned}
$$

where

$$
A_{\mu \nu}=-\frac{1}{S_{\mu \nu}}+\delta_{\mu \nu} \sum_{\xi} \frac{1}{S_{\mu \xi}}
$$

$S_{\mu \nu}$ is the length of the chain connecting junctions $\mu$ and $\nu$, and $1 / S_{\mu \nu}=0$ if junctions $\mu$ and $\nu$ are not connected.

The $y$ and $z$ distributions are equivalent to (2.2) and independent of $x$, and

$$
\begin{aligned}
S_{\mu \nu}^{\mathrm{eff}}=\left\langle\left(x_{\mu}-x_{\nu}\right)^{2}\right\rangle & = \\
& =\left(A^{-1}\right)_{\mu \mu}+\left(A^{-1}\right)_{\nu \nu}-2\left(A^{-1}\right)_{\mu \nu} .
\end{aligned}
$$

It has been noted by many authors that these equations are equivalent to Kirchhoff's equations at the junctions of a network of resistors. Eichinger [7, 10] uses Kirchhoff matrices and the formalism of graph theory to discuss the conformations of combinations of Gaussian chains. De Gennes [11] also discusses the parallel between the conductivity of a resistor network and the elastic modulus of a polymer system. We explicitly state the analogy below.

Consider a network of resistors which has the same connectivity as the polymer network. Each subchain $S_{\mu \nu}$ is represented by a resistor $\Omega_{\mu \nu}$. A current $I_{\mu}$ may enter each junction from outside. Kirchhoff's laws applied at the junctions give :

$$
I_{\mu}=M_{\mu \xi} V_{\xi}
$$

with

$$
M_{\mu \nu}=-\frac{1}{\Omega_{\mu \nu}}+\delta_{\mu \nu} \sum_{\xi} \frac{1}{\Omega_{\mu \xi}} .
$$

The point to point resistance $\Omega_{\mu \nu}^{\text {eff }}$ when current is allowed to flow through all possible routes between the junctions is given by

$$
\Omega_{\mu \nu}^{\text {eff }}=V_{\mu}-V_{\nu} \text { when } I_{\mu}=1, I_{\nu}=-1,
$$

and

$$
\begin{aligned}
& I_{\xi}=0 \text { for } \xi \neq \mu \text { or } \nu . \\
& \quad \Omega_{\mu \nu}^{\text {eff }}=\left(M^{-1}\right)_{\mu \mu}+\left(M^{-1}\right)_{\nu \nu}-2\left(M^{-1}\right)_{\mu \nu} .
\end{aligned}
$$

Thus if

$$
\Omega_{\mu \nu}=S_{\mu \nu} \text { then } S_{\mu \nu}^{\text {eff }}=\Omega_{\mu \nu}^{\text {eff }} .
$$

Clearly the result holds for any two points in the network, not merely junctions, since the points may be designated as junctions with only two subchains emerging from them. It is now possible to make progress in determining the point to point network fluctuations from the resistor analogy.

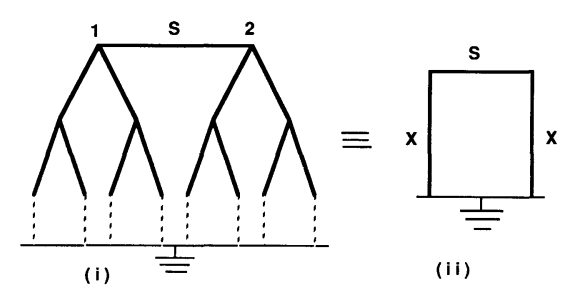

Fig. 1. - i) A network may be represented as a circuit with two infinite trees of resistors - shown here for functionality 3 ; ii) each of the trees may be replaced by a single resistance to ground $X$.

2. 2 The Bethe LATtice MODEL. - Figure $1 \mathrm{i}$ shows the circuit representation of the network if the tree approximation (discussed above) is made. It is a Bethe lattice with branching ratio $\alpha=\phi-1$.

Many systems have been approximated by Bethe lattices, since they give rise to recursion relations which often allow an «exact " solution of the problem. The review by Thorpe [12] discusses many examples. Stinchcombe [13] considered the conductivity of a Bethe lattice and derived relations equivalent to (2.8) and (2.9). The case of percolation was then treated in detail, but solutions of relevance to the present discussion were not obtained. Bethe lattices were also used in the work on phantom networks by Graessley [8].

The motion of two points in the network which are connected by a route passing through a small number of subchains will be correlated. Points which are connected only by routes passing through many subchains will have negligible correlation. For the Bethe lattice model to apply the only closed circuits in the network must be «large » in the sense that the correlations between opposite sides of the loop are negligible. We will obtain an estimate as to how large this has to be below.

Consider the resistor network of (2.6) represented by a Bethe lattice and focus on one particular resistor $S$ (Fig. 1). The value of $S$ has been chosen according to some (given) distribution of subchain lengths $f(S)$. The ends of this resistor are connected to earth by two infinite trees of resistors. Each of the resistors in the trees has a resistance independent of the others, also determined according to $f(S)$. The trees may be replaced by single resistances $X_{1}$ and $X_{2} . X_{1}$ is the resistance between junction 1 and earth if no current is allowed to flow through $S$. $S^{\text {eff }}$ is the resistance between junctions 1 and 2 if current is 
allowed to flow directly through $S$ and via earth through $X_{1}$ and $X_{2}$,

$$
\text { i.e. } \quad S^{\mathrm{eff}}=\frac{S\left(X_{1}+X_{2}\right)}{S+X_{1}+X_{2}}
$$

The values of $X_{1}$ and $X_{2}$ are governed by a probability distribution $g(X)$ which is dependent on $f(S)$ and which we wish to calculate. To do this we note that all the other junctions in the network are in principle no different from 1 and 2 . The resistance from any junction in the tree « downwards » to earth is a value of $X$ determined by the same probability distribution $g(X)$. The circuits in figure 2 are thus equivalent, and

$$
\frac{1}{X}=\sum_{i=1}^{\alpha} \frac{1}{S_{i}+X_{i}} .
$$

The sum is from 1 to $\alpha$ since there are $\alpha=\phi-1$ branches emerging downwards from each junction. We require the probability distribution of the new value $X$ to be the same as that of the $X_{i}$. Thus $g(X)$ is the solution of the self consistency relation :

$$
\begin{aligned}
g(X)=\int \ldots & \int \prod_{i=1}^{\alpha} \mathrm{d} S_{i} \mathrm{~d} X_{i} f\left(S_{i}\right) g\left(X_{i}\right) \times \\
& \times \delta\left(X-\left(\sum_{i=1}^{\alpha} \frac{1}{S_{i}+X_{i}}\right)^{-1}\right) .
\end{aligned}
$$

Analytical solutions for $g(X)$ have not been found for non trivial choices of $f(S)$. The relation has been solved numerically in section 3 , however several approximate solutions are possible, and are discussed here.

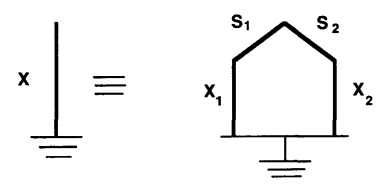

Fig. 2. - These two circuits are equivalent since each junction in the tree of fig. $1 \mathrm{i}$ is equivalent. This leads to the self-consistency relation (2.9).

2. 3 Approximate solutions. - The simplest approximation is to replace all the $S_{i}$ by their mean value $S_{0}$, and all the $X$ by a single value $X$, i.e. we assume that the network is monodisperse. (2.8) now becomes

$$
\frac{1}{X}=\frac{\alpha}{S_{0}+X}
$$

from which

$$
X=\frac{S_{0}}{\alpha-1}=\frac{S_{0}}{\phi-2} .
$$

We refer to this value of $X$ as $X_{\text {mono }}$ when we require to distinguish it. Substituting this into (2.7) we obtain :

$$
S^{\mathrm{eff}}=\frac{2 S_{0} X}{S_{0}+2 X}=\frac{2}{\phi} S_{0}
$$

which proves (1.5) for the monodisperse network.

A better approximation is to allow $S$ to take its full range of values, but to assume that the $X$ distribution can be characterized by a single mean field value $X_{\mathrm{M}}$. This value $X_{\mathrm{M}}$ will not be equal to $X_{\text {mono }}$, neither will it equal $X_{0}$, the mean of the true distribution $g(X)$. In the mean field approximation (2.8) becomes :

$$
\frac{1}{X_{\mathrm{M}}}=\alpha\left\langle\frac{1}{X_{\mathrm{M}}+S}\right\rangle_{s} \equiv \alpha \int_{0}^{\infty} \mathrm{d} S \frac{f(S)}{X_{\mathrm{M}}+S}
$$

The simplest non trivial distribution for $f(S)$, and one which is of experimental interest, is the « bimodal $»$ distribution: the network contains a fraction $p_{1}$ of short subchains of length $S_{1}$, and $p_{2}$ of long subchains of length $S_{2}$ connected randomly.

$$
f(S)=p_{1} \delta\left(S-S_{1}\right)+p_{2} \delta\left(S-S_{2}\right) .
$$

In this case :

$$
\frac{1}{X_{\mathrm{M}}}=\frac{\alpha p_{1}}{S_{1}+X_{\mathrm{M}}}+\frac{\alpha p_{2}}{S_{2}+X_{\mathrm{M}}} .
$$

This equation has one positive root $X_{\mathrm{M}}$. Table I compares the values of $X_{\mathrm{M}}$ with the true means $X_{0}$ calculated in section 3 , and with $X_{\text {mono }}$. For $S_{2} / S_{1}=2$, a moderately broad distribution for $f(S)$, it can be seen that the three values all lie within $\sim 5 \%$ of each other. There is however considerable disagreement in the more extreme case $S_{2} / S_{1}=20$.

The reason that the simple approximations work better than we might have expected is that the distribution $g(X)$ is much narrower than $f(S)$, as is discussed below and in appendix B.

Before using these values of $X$ to calculate experimentally measurable quantities it is useful to define some further functions of $S$ and $X$. The sum of two independent $X$ values occurs frequently (Eq. (2.7)), therefore we introduce a variable $Z=X_{1}+X_{2}$ with probability distribution :

$h(Z)=\iint \mathrm{d} X_{1} \mathrm{~d} X_{2} g\left(X_{1}\right) g\left(X_{2}\right) \delta\left(Z-X_{1}-X_{2}\right)$.

We denote the average appearing in the front factor (1.8) as $J$.

$$
J=\left\langle\frac{S^{\text {eff }}}{S}\right\rangle_{\text {all subchains }}=\left\langle\frac{Z}{S+Z}\right\rangle_{s, Z} .
$$


Table I. - The mean $X_{0}$ of the calculated $X$ distribution compared to the monodisperse and the mean field approximations for the bimodal networks defined in section 3.

\begin{tabular}{cccc}
$p_{1}: p_{2}$ & $X_{\text {mono }}$ & $X_{\mathrm{M}}$ & $X_{0}$ \\
& SERIES 1 & $-S_{2} / S_{1}=2$ & \\
$0: 100$ & 2.000 & - & - \\
$10: 90$ & 1.900 & 1.869 & $1.884 \pm 0.001$ \\
$30: 70$ & 1.700 & 1.628 & 1.661 \\
$50: 50$ & 1.500 & 1.414 & 1.451 \\
$70: 30$ & 1.300 & 1.128 & 1.257 \\
$90: 10$ & 1.100 & 1.070 & 1.080 \\
$100: 0$ & 1.000 & - & - \\
\multicolumn{5}{c}{ SERIES 2} & $-S_{2} / S_{1}=20$ & \\
$0: 100$ & 20.00 & - & - \\
$10: 90$ & 18.10 & 16.42 & $17.38 \pm 0.01$ \\
$30: 70$ & 14.30 & 9.67 & 12.23 \\
$50: 50$ & 10.50 & 4.47 & 7.36 \\
$70: 30$ & 6.70 & 2.07 & 3.51 \\
$90: 10$ & 2.90 & 1.22 & 1.45 \\
$100: 0$ & 1.00 & &
\end{tabular}

For the bimodal distribution this reduces to the weighted sum of two one dimensional integrals, $J_{1}$ and $J_{2}$.

$$
\begin{aligned}
J & =p_{1}\left\langle\frac{Z}{S_{1}+Z}\right\rangle_{Z}+p_{2}\left\langle\frac{Z}{S_{2}+Z}\right\rangle_{Z} \\
& =p_{1} J_{1}+p_{2} J_{2} .
\end{aligned}
$$

It is $J_{1}$ and $J_{2}$ which determine the way the short and long chains extend when the network is stretched (see section 4). We can define mean field values for $Z, J_{1}, J_{2}$ and $J$ in an obvious way.

$$
\begin{gathered}
Z_{\mathrm{M}}=2 X_{\mathrm{M}} \\
J_{1}^{\mathrm{M}}=\frac{Z_{\mathrm{M}}}{S_{1}+Z_{\mathrm{M}}} \quad J_{2}^{\mathrm{M}}=\frac{Z_{\mathrm{M}}}{S_{2}+Z_{\mathrm{M}}} \\
J_{\mathrm{M}}=p_{1} J_{1}^{\mathrm{M}}+p_{2} J_{2}^{\mathrm{M}} .
\end{gathered}
$$

We note that while $J$ is identically $2 / \phi$ for all $f(S), J_{\mathrm{M}}$ is only approximately $2 / \phi$. The extent of this error in the mean field approximation can be seen from table II. It is again only large for the very broad distributions $S_{2} / S_{1}=20$.

A very good approximation to the self-consistent distribution $g(X)$ can be obtained when $f(S)$ is itself a narrow distribution. If we write

$$
S=S_{0}+s \text { and } X=X_{0}+x
$$

\begin{tabular}{|c|c|c|c|c|c|c|}
\hline \multicolumn{4}{|c|}{ Calculated valucs } & \multicolumn{3}{|c|}{ Mean field values } \\
\hline$p_{1}: p_{2}$ & $J$ & $J_{1}$ & $J_{2}$ & $J^{\mathrm{M}}$ & $J_{1}^{\mathrm{M}}$ & $J_{2}^{\mathrm{M}}$ \\
\hline \multicolumn{7}{|c|}{ SERIES $1-S_{2} / S_{1}=2$} \\
\hline $0: 100$ & - & - & - & 0.667 & 0.800 & 0.667 \\
\hline $10: 90$ & $0.667(1)$ & $0.790(1)$ & $0.653(1)$ & 0.665 & 0.789 & 0.652 \\
\hline $30: 70$ & 0.667 & 0.768 & 0.623 & 0.663 & 0.765 & 0.620 \\
\hline $50: 50$ & 0.667 & 0.742 & 0.591 & 0.662 & 0.739 & 0.586 \\
\hline $70: 30$ & 0.667 & 0.714 & 0.556 & 0.663 & 0.711 & 0.551 \\
\hline $90: 10$ & 0.666 & 0.683 & 0.519 & 0.665 & 0.682 & 0.519 \\
\hline $100: 0$ & - & - & - & 0.667 & 0.667 & 0.500 \\
\hline \multicolumn{7}{|c|}{ SERIES $2-S_{2} / S_{1}=20$} \\
\hline $0: 100$ & - & - & - & 0.67 & 0.98 & 0.67 \\
\hline $10: 90$ & $0.67(1)$ & $0.97(1)$ & $0.63(1)$ & 0.66 & 0.97 & 0.62 \\
\hline $30: 70$ & 0.67 & 0.96 & 0.54 & 0.63 & 0.95 & 0.49 \\
\hline $50: 50$ & 0.67 & 0.93 & 0.41 & 0.60 & 0.90 & 0.31 \\
\hline $70: 30$ & 0.67 & 0.85 & 0.24 & 0.62 & 0.80 & 0.17 \\
\hline $90: 10$ & 0.66 & 0.72 & 0.12 & 0.65 & 0.71 & 0.11 \\
\hline $100: 0$ & - & - & - & 0.67 & 0.67 & 0.09 \\
\hline
\end{tabular}

so that

$$
\langle s\rangle=\langle x\rangle=0
$$

Table II. - Calculated values of the integrals $J$, $J_{1}$, and $J_{2}$ compared to the mean field approximation for the bimodal networks discussed in section 3.

then we may expand the factor $\left(\sum_{i} \frac{1}{S_{i}+X_{i}}\right)^{-1}$ occurring in (2.9) in powers of $s$ and $x$. Quantities of interest may be written as series in the moments $\left\langle s^{2}\right\rangle,\left\langle s^{3}\right\rangle \ldots$ These series will rapidly converge when $f(S)$ has a narrow spread about $S_{0}$. The details are given in appendix B. Figure 3 shows $g(X)$ calculated by the moments expansion compared to the full self-consistent solution, in the case of the very narrow bimodal distribution :

$$
f(S)=0.5 \delta(S-1.00)+0.5 \delta(S-1.05) .
$$

The approximation shows all the significant features of the distribution. An important result of the appendix is that :

$$
\left\langle x^{2}\right\rangle=\frac{\left\langle s^{2}\right\rangle}{\alpha^{3}-1}+O\left(\left\langle s^{3}\right\rangle\right) .
$$

Thus, as stated before, the $X$ distribution is much narrower than the $S$ distribution. This is particularly true for high functionality. We would expect the mean field solution to become better as $\phi$ increases. This is useful because obtaining the self-consistent solution becomes very time consuming for large $\phi$.

\section{The self consistent solution obtained numerically.}

We now solve (2.9) numerically for several functions $f(S)$ of interest. The method is to use a trial function $g(X)$ on the right of the equation, evaluate the integral to give a new function $g^{\prime}(X)$, then use this as a new trial function. The procedure is repeated until $g^{\prime}(X)=g(X)$. The process can be viewed as choosing the values of the resistances many layers down 


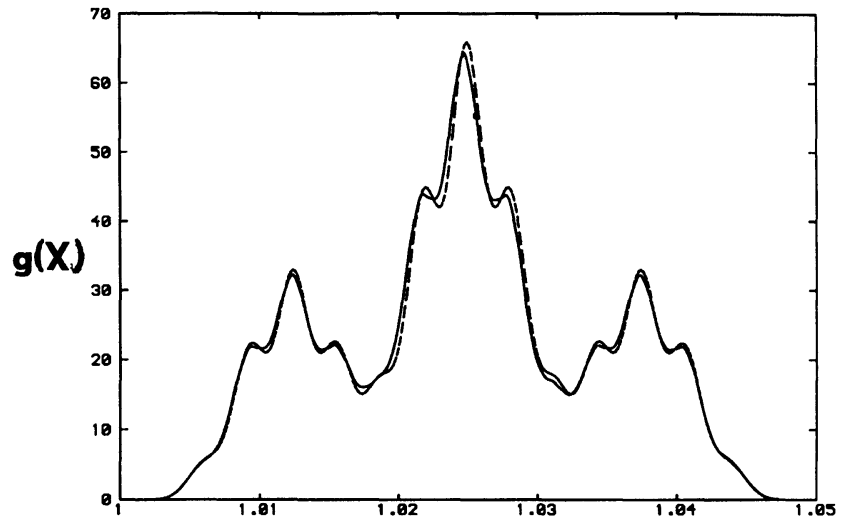

Fig. 3. - The probability distribution function $g(X)$ of resistance to ground calculated for a functionality 3 bimodal network with $50 \%$ chains length 1.00 and $50 \%$ chains length 1.05 . Solid line : self-consistent solution. Broken line : approximation using moments expansion. The $1: 2: 1$ ratio of heights of the three main peaks is due to the three possible combinations of the two resistances in the top layer of the tree.

the tree in figure 1 according to the initial trial function, and on each iteration moving one layer up the tree. We reach the top of the tree on convergence of the distribution $g(X)$. The values of the resistances in the lower layers of the tree have very little effect on the value of the total $X$. In other words the process will converge to a self-consistent solution independently of the choice of the initial trial.

Choice of a suitable initial trial function will increase the rapidity of convergence. When $f(S)$ is a bimodal distribution there is a finite maximum and minimum value of $X$ which occur when all the resistors in the tree are of one type.

$$
X_{\min }=\frac{S_{1}}{\phi-2} \quad X_{\max }=\frac{S_{2}}{\phi-2} .
$$

For the results in table II we use a uniform « top hat " distribution between $X_{\min }$ and $X_{\max }$ as the trial. This interval is divided into $N$ equally sized bins with discrete probabilities $g_{n}$ assigned to each bin $n$. All calculations in this section deal with $\phi=3$ for ease of computation.

The program was tested with $N=200$ using the narrow bimodal distribution (2.18). The same solution was obtained from three different trials. The iterations were stopped when typical values of the probabilities $g_{n}$ had remained constant to at least 5 significant figures for several iterations. In each case convergence occurred after about 20 iterations, i.e. the correlation between points $\geq 20$ subchains apart is negligible. This gives us an indication of the size of loops which can be tolerated. Experimental measurements are likely to be considerably less precise than this, and to ensure convergence to 3 significant figures only 6-8 iterations were required. (We cannot directly equate the number of iterations with the loop size since to close a loop requires a short path to earth from both ends of a subchain. This does however provide a reasonable estimate.)

The shape of the curve in figure 3 is easily understandable. The resistance to earth is most strongly dependent on the resistors at the top of the tree. There are three possible combinations of resistors in the top layer of the tree: $S_{1} S_{1}$, $S_{1} S_{2}$, and $S_{2} S_{2}$. These occur in a $1: 2: 1$ ratio, hence the three main peaks in figure 3 . These peaks are each split into three due to the values of the resistors in the second layer. Further structure is not seen. The $g(X)$ distributions only have the near symmetry of figure 3 when $f(S)$ is very narrow, cf. figure 4 .

Two series of distributions were calculated with $S_{1}$ and $S_{2}$ fixed and varying $p_{1}$ and $p_{2}$.

$$
\begin{array}{lll}
\text { Series } 1-S_{1}=1.0 & S_{2}=2.0 & N=300 \\
\text { Series } 2-S_{1}=1.0 & S_{2}=20.0 & N=200 .
\end{array}
$$

An estimate of the accuracy of the method can be obtained by looking at the values of $J$ in table II, which should of course be exactly $2 / 3$. The error is due to the discretization of the probability distribution. If sufficient bins are used the method is exact.

The other $f(S)$ distribution of interest is the Poisson distribution, which occurs if we assume every monomer on the original chain has an equal chance $p$ of becoming a crosslink. The probability of a subchain having $n$ monomers is thus

$$
f(n)=(1-p)^{n-1} p \approx \frac{1}{n_{0}} \exp \left(-n / n_{0}\right)
$$

when $p \rightarrow 0 \quad\left(n_{0}=1 / p\right)$

hence

$$
f(S)=\frac{1}{S_{0}} \exp \left(-S / S_{0}\right)
$$

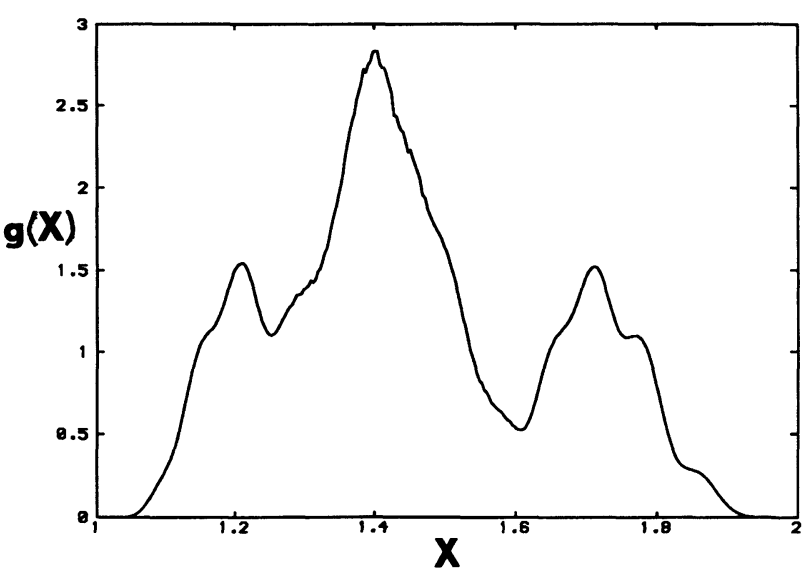

Fig. 4. - Self consistent solution for $g(X)$ for a functionality 3 bimodal network with $50 \%$ chains length 1 and $50 \%$ chains length 2 . The function no longer has the near-symmetry of figure 3 when the input $S$ distribution is broader. 
Since both the $S$ and the $X$ distributions are continuous there are $\mathrm{O}\left(N^{4}\right)$ combinations of the four resistances instead of $\mathrm{O}\left(N^{2}\right)$ as before, so it is necessary to use a much smaller number of bins. Here $X_{\min }$ is zero and we introduce a cutoff in the $S$ and the $X$ distributions at $S_{\mathrm{c}}$ beyond which the probability is taken to be zero. The results in figure 5 were obtained using 36 bins of widths which were smallest at small $S$ in order to obtain the maximum resolution of the distributions in the region where they have their greatest weight. The mean of this distribution is found to be $X_{0}=0.77 \pm 0.01$, and the distribution yields a value of $J=0.67 \pm 0.01$. This is to be compared with $X_{\text {mono }}=1.0$ and $X_{\mathrm{M}}=0.610$. The mean field approx in the Poisson case is

$$
\begin{gathered}
\frac{1}{X_{\mathrm{M}}}=\alpha \int_{0}^{\infty} \frac{\mathrm{e}^{-s}}{S+X_{\mathrm{M}}} \mathrm{d} S \\
J_{\mathrm{M}}=\int_{0}^{\infty} \frac{2 X_{\mathrm{M}} \mathrm{e}^{-s}}{2 X_{\mathrm{M}}+S} \mathrm{~d} S .
\end{gathered}
$$

Numerical solutions for $X_{\mathrm{M}}$ and $J_{\mathrm{M}}$ are shown for several values of $\phi$ in table III. We note from figure 5 that $g(X)$ is quite sharply peaked and dies off much more rapidly than $f(S)$ for large $X$, and that the mode of the distribution is approximately at $X_{\mathrm{M}}$.

Table III. - Values of $X_{\mathrm{M}}$ and $J_{\mathrm{M}}$ for networks with Poisson distributions of subchain lengths with various functionalities compared to the mean of the calculated distribution for $\phi=3$ and the true $J$ values $(=2 / \phi)$.

$\begin{array}{ccccc}\phi & X_{0} & X_{\mathrm{M}} & J_{\mathrm{M}} & 2 / \phi \\ 3 & 0.77 \pm 0.01 & 0.610 & 0.634 & 2 / 3 \\ 4 & & 0.247 & 0.459 & 1 / 2 \\ 6 & & 0.0988 & 0.298 & 1 / 3\end{array}$

\section{The elasticity of bimodal networks.}

Bimodal networks are of particular interest because they are the simplest polydisperse network and because of the model networks of Poly-dimethylsiloxane studied by Mark et al. [5]. These experiments have shown that the stress strain curves for the networks are very dependent on the ratio $p_{1}: p_{2}$ of short to long chains, and that both the stress and strain required to break the sample are

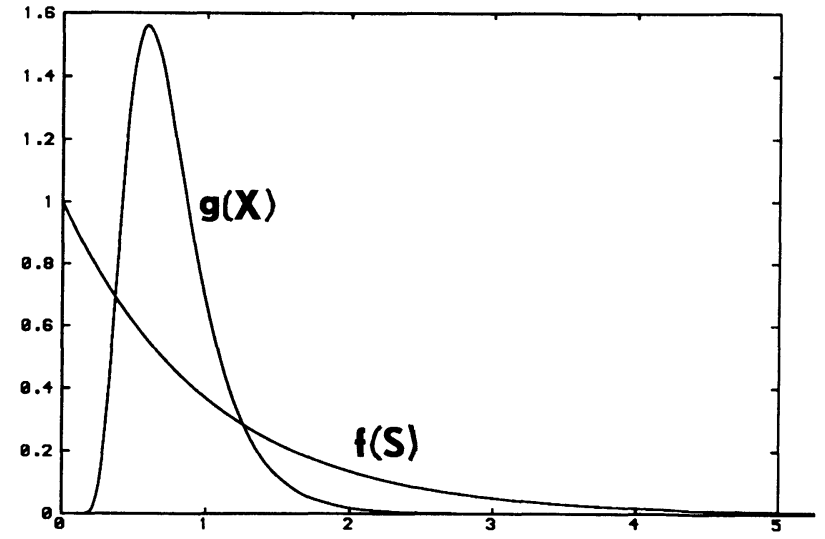

Fig. 5. - The subchain length distribution $f(S)=\mathrm{e}^{-S}$ and the self-consistent solution for $g(X)$ for a functionality 3 network with randomly positioned crosslinks (radiative crosslinking). Despite the slow tail-off of $f(S), g(X)$ tails off rapidly for large $X$ and is strongly peaked about a characteristic value. The peak will be sharper for higher functionality.

often much higher for the mixed networks than for networks of either the long or the short chains alone.

These results strongly disagree with the assertion of section 1 that the elastic properties should be independent of the subchain length distribution. This is directly attributable to the non Gaussian nature of the very short chains in the experimental materials and their limited extensibility. Curro and Mark [14, 15] have shown using rotational isomeric state models that the short chains in their experiments have an end to end vector distribution significantly different from a Gaussian. A detailed interpretation of these experiments is thus outside the scope of this paper, however the present study of Gaussian networks will clarify some points concerning the response of the different chain species when the network is stretched which are of relevance to the experiments.

Before deformation

$$
p(\mathbf{r})=\frac{1}{(2 \pi S)^{3 / 2}} \exp \left(-r^{2} / 2 S\right)=p(x) p(y) p(z)
$$

where

$$
p(x)=\frac{1}{(2 \pi S)^{1 / 2}} \exp \left(-x^{2} / 2 S\right) .
$$

Upon a deformation along the principal axes $x$ becomes $x=\lambda_{x} R_{x}+\rho_{x}$, and $p(x)$ becomes :

$$
\begin{aligned}
p_{\lambda}(x) & =\iint \mathrm{d} R_{x} \mathrm{~d} \rho_{x} \frac{1}{\left(2 \pi S^{\text {eff }}\right)^{1 / 2}} \frac{1}{\left(2 \pi\left(S-S^{\text {eff }}\right)\right)^{1 / 2}} \exp \left(-\frac{R^{2}}{2\left(S-S^{\text {eff }}\right)}\right) \exp \left(-\frac{\rho^{2}}{2 S^{\text {eff }}}\right) \delta\left(x-\lambda_{x} R_{x}-\rho_{x}\right) \\
& =\frac{1}{\left(2 \pi L_{x}\right)^{1 / 2}} \exp \left(-\frac{x^{2}}{2 L_{x}}\right)
\end{aligned}
$$


where

$$
\begin{gathered}
L_{x}=\lambda_{x}^{2}\left(S-S^{\mathrm{eff}}\right)+S^{\mathrm{eff}} \\
\left\langle r^{2}\right\rangle_{\lambda}=L_{x}+L_{y}+L_{z}=\sum_{i} \lambda_{i}^{2}\left(S-S^{\mathrm{eff}}\right) \\
+3 S^{\mathrm{eff}} \\
=\frac{1}{3} \sum_{i} \lambda_{i}^{2}\left\langle R^{2}\right\rangle_{0}+\left\langle\rho^{2}\right\rangle_{0} \\
\frac{\left\langle r^{2}\right\rangle_{\lambda}}{\left\langle r^{2}\right\rangle_{0}}=\frac{1}{3} \sum_{i} \lambda_{i}^{2}\left(1-J_{s}\right)+J_{s} .
\end{gathered}
$$

This ratio has a different value for the short subchains $\left(J_{s}=J_{1}\right)$ and the long subchains $\left(J_{s}=J_{2}\right)$. Since $J_{2}<J_{1}$ it is the long chains which are more extended relative to their initial length than the short chains. We will consider the uniaxial extension $\lambda_{z}=\lambda, \lambda_{x}=\lambda_{y}=\lambda^{-1 / 2}$.

The radial distribution function before deformation is given by

$P(r)=4 \pi r^{2} p(r)=\left(\frac{2}{\pi S^{3}}\right)^{1 / 2} r^{2} \exp \left(-r^{2} / 2 S\right)$.

After deformation we may express the $\mathbf{r}$ distribution in polar coordinates as

$$
\begin{aligned}
p_{\lambda}(\mathbf{r})=p_{\lambda}(x) p_{\lambda}(y) p_{\lambda}(z)=\left(\frac{1}{8 \pi^{3} L_{x}^{2} L_{z}}\right)^{1 / 2} \times \\
\times \exp \left(-\frac{r^{2} \sin ^{2} \Theta}{2 L_{x}}-\frac{r^{2} \cos ^{2} \Theta}{2 L_{z}}\right) .
\end{aligned}
$$

Now integrating w.r.t. $\Theta$ and $\Phi$ we obtain the new radial distribution function

$$
\begin{aligned}
P_{\lambda}(r)= & \int_{0}^{2 \pi} \mathrm{d} \Phi \int_{0}^{\pi} \mathrm{d} \Theta r^{2} \sin \Theta p_{\lambda}(\mathbf{r}) \\
= & \frac{2 r^{2}}{\left(\pi L_{x}\left(L_{z}-L_{x}\right)\right)^{1 / 2}} \times \\
& \times \exp \left(-r^{2} / 2 L_{x}\right) D\left(r \sqrt{\frac{L_{z}-L_{x}}{L_{z} L_{x}}}\right)
\end{aligned}
$$

where $D$ is Dawson's integral $D(x)=\mathrm{e}^{-x^{2}} \int_{0}^{x} \mathrm{e}^{t^{2}} \mathrm{~d} t$.

$P_{\lambda}(r)$ can be evaluated directly in the monodisperse case since $L_{x}$ and $L_{z}$ have single values. In the bimodal case $L_{x}$ and $L_{z}$ are functions of $S$ and $Z$.

$$
\begin{aligned}
& L_{z}=\lambda^{2} S+\left(1-\lambda^{2}\right) \frac{S Z}{S+Z} \\
& L_{x}=\frac{S}{\lambda}+(1-1 / \lambda) \frac{S Z}{S+Z} .
\end{aligned}
$$

The radial distribution function for the short chains
$P_{\lambda}^{(1)}(r)$ is obtained by averaging over the $Z$ distribution with $S$ fixed at $S_{1}$.

$$
P_{\lambda}^{(1)}(r)=\left.\int \mathrm{d} Z h(Z) P_{\lambda}(r)\right|_{s=s_{1}} .
$$

Similarly for the long chain distribution $P_{\lambda}^{(2)}(r)$.

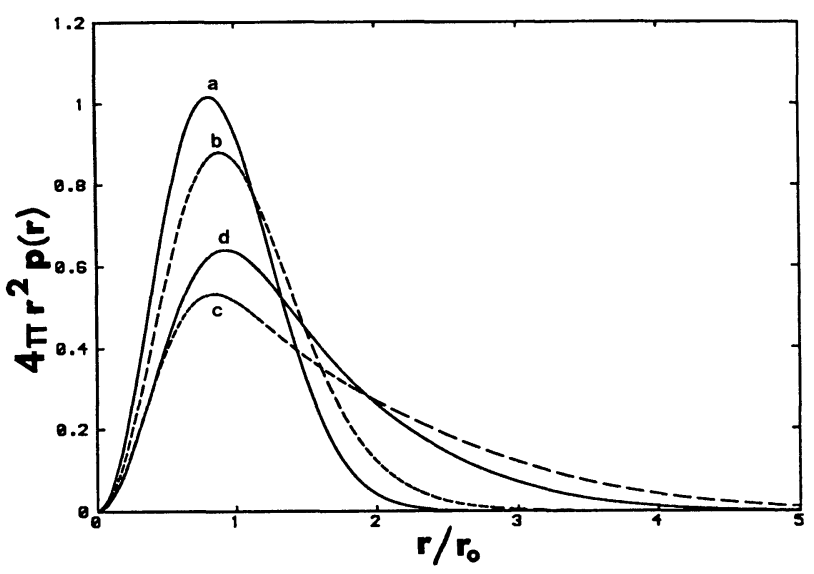

Fig. 6. - Radial distribution functions of chains in a functionality 3 bimodal network (50\% length $1,50 \%$ length 20). The $r$ scale is measured in terms of $r_{0}$, the initial r.m.s. length of the chain species in question. a) The unstrained radial distribution function, which with this scaling is the same for the short and long chains ; b) short chains at $\lambda=4$, c) long chains at $\lambda=4$. The long chains are much more extended than the short relative to their initial r.m.s. length; d) chains in a monodisperse network at $\lambda=4$.

Figure 6 shows $P_{\lambda}^{(1)}(r)$ and $P_{\lambda}^{(2)}(r)$ evaluated at $\lambda=4$ for the network with $S_{2} / S_{1}=20$ and $p_{1}=p_{2}=0.5$. The $r$ scale is measured in units of $r_{0}$, the initial root mean square length (which is of course different for the short and the long subchains). The curves are compared to the undeformed distribution, which with this scaling is the same for the short and the long subchains, and to $P_{\lambda}(r)$ for a monodisperse network at $\lambda=4$.

(Evidently a mean field version of $P_{\lambda}^{(1)}$ and $P_{\lambda}^{(2)}$ is possible, with $Z=Z_{\mathrm{M}}$ and no integration over $Z$. The curves are found to be almost indistinguishable from the self consistent solution when $S_{2} / S_{1}=2$ but in rather poor agreement when $S_{2} / S_{1}=20$.)

The stress strain curves for the model networks show a significant upturn at a deformation $\lambda_{\mathrm{u}}$ before the break point is reached, i.e. the modulus increases. This has been interpreted as due to the limited extensibility of the short relative to the long chains. Although the short chains are less extended than the long relative to their initial rms length $r_{0}=\sqrt{N \ell}$, they are more extended relative to their 
maximum length $r_{\mathrm{m}}=N \ell$. It is important to distinguish clearly between the two measures. Andrady, Llorente and Mark [16, 17] have compared typical values of $r$ at $\lambda_{\mathrm{u}}$ with $r_{\mathrm{m}}$ obtained from the rotational isomeric state model. Reasonable correlation was found. The modulus began to increase at $r / r_{\mathrm{m}} \sim$ $60-70 \%$.

Such comparisons are only of limited value since firstly the typical $r$ value chosen was not the value of the root mean square length predicted by the phantom network model (4.5), and also since choosing any single typical $r$ value will not give information on the extension of the few chains which have very large end to end distances. A better approach might be to consider the high $r$ end of the radial distribution functions. If a significant proportion of the chains are predicted by the Gaussian model to have end to end distances greater than $r_{\mathrm{m}}$ then clearly the model will not be valid and the modulus will be higher than predicted. It is the few chains with very large extensions which will first cause the increase in the modulus.

\section{Orientational properties of network chains.}

There are many experimental techniques for measuring the orientational order introduced into the monomer units in the chains when a network is stretched. Kuhn and Grun [18] showed that the birefringence of a strained network is proportional to $\left\langle P_{2}(\cos \chi)\right\rangle$ (where $\chi$ is the angle between a monomer unit and the direction of strain and $P_{2}$ is the second Legendre polynomial). $\left\langle P_{2}\right\rangle$ can also be measured by Fluorescence polarization, and broad line nuclear magnetic resonance yields measurements of $\left\langle P_{2}\right\rangle$ and $\left\langle P_{4}\right\rangle$. These and other techniques are reviewed by Ward [19].

We obtain values for $\left\langle P_{2}\right\rangle$ and $\left\langle P_{4}\right\rangle$ for polydisperse networks with particular reference once again to bimodal networks. In this case there is the possibility, by selective labelling of the network, of measuring the orientational order of one of the chain species independently of the other [20]. Monomer unit orientation in the monodisperse network has been treated by Treloar [21] and by Roe and Krigbaum [22]. We need only summarize the results and point out the differences due to polydispersity.

The calculation of the chain orientation is unusual in that the step length $\ell$ and number of steps $N$ are critical, since in the perfectly Gaussian limit $(\ell \rightarrow 0$ and $N \ell^{2}=$ constant) the monomer units would be isotropic at all strains. The polymer molecule is represented by a chain of freely hinged rods. A typical rod makes an angle $\theta$ with the end to end vector of the chain, and an angle $\chi$ with the $z$ axis (the direction of strain). The end to end vector $\mathbf{r}$ makes an angle $\Theta$ with the $z$ axis. We wish to calculate $\left\langle P_{2}(\xi)\right\rangle$ and $\left\langle P_{4}(\xi)\right\rangle$ where :

$$
\begin{aligned}
\xi & =\cos \chi \\
P_{2}(\xi) & =\frac{3}{2} \xi^{2}-\frac{1}{2} \\
P_{2}(\xi) & =\frac{35}{8} \xi^{4}-\frac{30}{8} \xi^{2}+\frac{3}{8} .
\end{aligned}
$$

It is found that the monomer units on any chain have a most probable distribution of orientations with respect to the end to end vector of that chain given by :

$$
f(\theta) \mathrm{d} \theta=\frac{1}{2} \frac{\beta}{\sinh \beta} \exp (\beta \cos \theta) \sin \theta \mathrm{d} \theta
$$

where $\beta$ is the inverse Langevin function $L^{-1}(r / N \ell)$ defined by :

$$
L(\beta)=\operatorname{coth} \beta-1 / \beta=r / N \ell=t .
$$

We may write $\beta$ as a power series in $t$, and evaluate the Legendre polynomials for small $t$. Following the method of Roe and Krigbaum we find, with the polynomials normalized as above,

$$
\begin{aligned}
& \left\langle P_{2}(\xi)\right\rangle=\int_{0}^{2 \pi} \mathrm{d} \Phi \int_{0}^{\pi} \mathrm{d} \Theta \int_{0}^{\infty} \mathrm{d} r r^{2} \sin ^{2} \Theta p_{\lambda}(\mathbf{r}) P_{2}(\cos \Theta)\left\{\frac{3}{5} t^{2}+\frac{36}{175} t^{4}+\mathrm{O}\left(t^{6}\right)\right\} \\
& \left\langle P_{4}(\xi)\right\rangle=\int_{0}^{2 \pi} \mathrm{d} \Phi \int_{0}^{\pi} \mathrm{d} \Theta \int_{0}^{\infty} \mathrm{d} r r^{2} \sin ^{2} \Theta p_{\lambda}(\mathbf{r}) P_{4}(\cos \Theta)\left\{\frac{3}{35} t^{4}+\mathrm{O}\left(t^{6}\right)\right\}
\end{aligned}
$$

where $p_{\lambda}(\mathbf{r})$ is the deformed vector distribution from (4.7). Evaluating (5.3) to leading order gives :

$$
\left\langle P_{2}(\xi)\right\rangle=\frac{\ell^{2}}{15}\left(\lambda^{2}-\frac{1}{\lambda}\right) \frac{\left(S-S^{\mathrm{eff}}\right)}{S^{2}} .
$$

In the monodisperse case, putting $S=N \ell^{2} / 3$, this becomes :

$$
\left\langle P_{2}(\xi)\right\rangle=\frac{1}{5 N}\left(\lambda^{2}-\frac{1}{\lambda}\right)\left(1-\frac{2}{\phi}\right) .
$$


This is the result obtained by Erman and Flory [23]. It differs from the original result of [18, 21, 22] by the factor $(1-2 / \phi)$, the same factor which occurs in the modulus.

In the polydisperse case we need to take the average of $\left(S-S^{\text {eff }}\right) / S^{2}$ weighting all monomers equally rather than weighting all subchains equally as before, i.e. there is an extra factor of $S / S_{0}$ in the probability distribution.

$$
\begin{aligned}
\left\langle\frac{S-S^{\mathrm{eff}}}{S^{2}}\right\rangle_{\text {monomers }} & =\iint \mathrm{d} S \mathrm{~d} Z \frac{S f(S)}{S_{0}} h(Z) \frac{1}{S}\left(1-\frac{Z}{S+Z}\right) \\
& =\frac{1}{S_{0}}(1-J)=\frac{1}{S_{0}}(1-2 / \phi) .
\end{aligned}
$$

Thus (5.6) applies for the polydisperse network too. The leading term in the birefringence remains proportional to the stress in the polydisperse case.

For completeness we obtain the $\mathrm{O}\left(t^{4}\right)$ in $P_{2}$.

$$
\mathrm{O}\left(t^{4}\right) \text { term }=\frac{2 l^{2}}{9} \frac{1}{175}\left\{\left(6 \lambda^{2}+2 \lambda-\frac{8}{\lambda}\right) \frac{\left(S-S^{\mathrm{eff}}\right)^{2}}{S^{4}}+14\left(\lambda^{2}-\frac{1}{\lambda}\right) \frac{S^{\mathrm{eff}}\left(S-S^{\mathrm{eff}}\right)}{S^{4}}\right\} .
$$

The original treatment had the junction points deforming affinely. This is equivalent to the $\phi=\infty$ limit of the phantom network model, where $S^{\text {eff }}=0$. In the monodisperse junction affine case :

$$
\mathrm{O}\left(t^{4}\right) \text { term }=\frac{2}{175 N^{2}}\left(6 \lambda^{4}+2 \lambda-\frac{8}{\lambda^{2}}\right) .
$$

This is given by Treloar. To leading order $P_{4}$ is given by :

$$
\begin{aligned}
\left\langle P_{4}(\xi)\right\rangle & =\frac{l^{4}}{9} \frac{1}{35}\left(\lambda^{2}-\frac{1}{\lambda}\right)^{2} \frac{\left(S-S^{\mathrm{eff}}\right)^{2}}{S^{4}} \\
& =\frac{1}{35 N^{2}}\left(\lambda^{2}-\frac{1}{\lambda}\right)^{2}(1-2 / \phi)^{2} \text { in the monodisperse case }
\end{aligned}
$$

Unlike $P_{2}, P_{4}$ is dependent on $f(S)$. (Both the $\mathrm{O}\left(t^{4}\right)$ terms appear to differ from Roe and Krigbaum by a factor of $5 / 3$.)

We express the results for the distributions considered in section 3 as a ratio of $P_{n}$ for the polydis- perse network to $P_{n}$ for the monodisperse network with subchain length equal to the length of the short chains. We consider leading order only. For the bimodal networks we define :

$$
\begin{aligned}
A_{1}=\frac{\left\langle P_{2}\right\rangle_{\text {short chains }}}{\left\langle P_{2}\right\rangle_{\text {mono. short }}}=\frac{\left(1-J_{1}\right)}{(1-2 / \phi)} ; \\
A_{2}=\frac{\left\langle P_{2}\right\rangle_{\text {long chains }}}{\left\langle P_{2}\right\rangle_{\text {mono. short }}}=\frac{S_{1}\left(1-J_{2}\right)}{S_{2}(1-2 / \phi)} ; \\
A=\frac{\left\langle P_{2}\right\rangle_{\text {whole network }}}{\left\langle P_{2}\right\rangle_{\text {mono. short }}}=\frac{1}{S_{1}}\left(p_{1} S_{1} A_{1}+p_{2} S_{2} A_{2}\right)=1 .
\end{aligned}
$$


Treating the data for the fourth polynomial in the same way,

$$
\begin{aligned}
B_{1} & =\frac{\left\langle P_{4}\right\rangle_{\text {short chains }}}{\left\langle P_{4}\right\rangle_{\text {mono. short }}} \\
& =\frac{S_{1}^{2}}{(1-2 / \phi)^{2}} \int \mathrm{d} Z \frac{1}{\left(S_{1}+Z\right)^{2}} h(Z) ;
\end{aligned}
$$

similarly

$$
B_{2}=\frac{\left\langle P_{4}\right\rangle_{\text {long chains }}}{\left\langle P_{4}\right\rangle_{\text {mono. short }}} ;
$$

and

$$
B=\left(p_{1} S_{1} B_{1}+p_{2} S_{2} B_{2}\right) / S_{1}
$$

which is not in general equal to 1 .
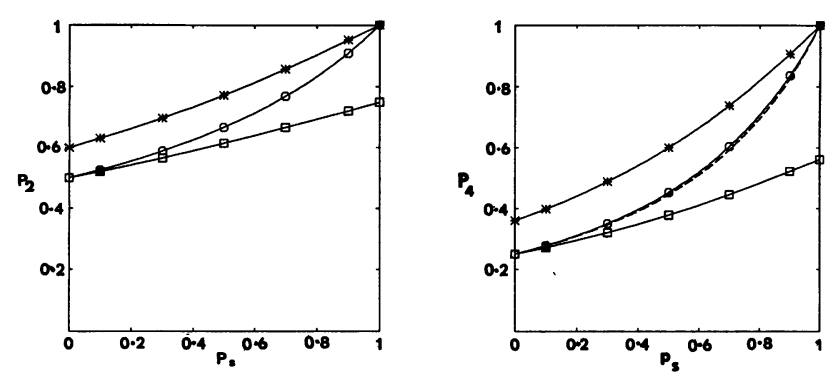

Fig. 7. - Chain orientation in a bimodal network (50\% length $1,50 \%$ length 2 ). The Legendre polynomials $\left\langle P_{2}\right\rangle$ and $\left\langle P_{4}\right\rangle$ calculated as the ratio of short to long chains is altered ( $p_{s}$ is three fraction of short chains). The vertical scales show relative values. Absolute values depend on the number of statistical segments in the chains. $O$ Average over all chains, * Short chains only; $\square$ Long chains only. The broken line is $\left\langle P_{4}\right\rangle$ for a monodisperse network with the same mean subchain length.

Figures 7 and 8 show these functions plotted against $p_{1}$, the proportion of short chains. The difference between the orientation of the monomers in the short and the long chains can be seen to be large in all cases. The results reinforce the fact that the short chains are more extended relative to their maximum length, therefore $A_{1}>A_{2}$, but less extended relative to their initial rms length.

Using the calculated $g(X)$ for the Poisson distribution with $\phi=3$ we find that the ratios of $\left\langle P_{2}\right\rangle$ and $\left\langle P_{4}\right\rangle$ relative to a monodisperse network with the same mean subchain length are $A=0.99 \pm 0.01$ and $B=1.13 \pm 0.01$. A would of course be exactly 1 if there were no inaccuracies in
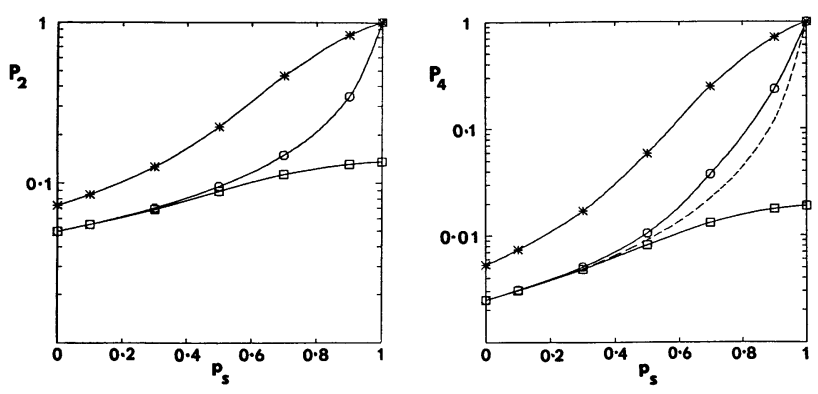

Fig. 8. - As figure 7 for a network with $50 \%$ length 1 , $50 \%$ length 20 . Note the logarithmic scale. The orientation of the short chains is an order of magnitude greater than the long chains.

the calculation, whereas the deviation of $B$ from 1 is real.

Erman and Flory [23] have considered some complications which are likely to occur in real networks, such as the restriction in junction fluctuations which will be caused by entanglements and dense chain packing. They have predicted that the stress : birefringence ratio will actually depend on the strain. They have some success in matching experimental results to their theory. However, the differences between $A_{1}$ and $A_{2}$ predicted here are so large as to make them easily experimentally observable, and the phantom network calculations provide a reasonable basis for comparison. Mean field predictions for $A_{1}$ and $A_{2}$ can easily be obtained by substituting the values $J_{1}^{\mathrm{M}}$ and $J_{2}^{\mathrm{M}}$ in (5.10). In view of the other complications which would occur in a real system it is thought that the mean field approximation would be a sufficiently accurate starting point, especially since real systems are likely to be $\phi=4$ rather than $\phi=3$.

In recent work by Mitchell [24] values for $P_{2}$ are obtained from wide angle $\mathrm{X}$ ray scattering. The results highlight the fact that the theory is expected to apply only for $r^{2}<(N \ell)^{2}$ i.e. for $\lambda^{2}<N$. In these experiments values of $N$ required to give the measured $P_{2}$ values are $\sim \lambda^{2}$. Terms of higher order in $t$ are important in this case.

\section{SANS from polymer networks.}

The results of recent neutron scattering experiments (to be discussed below) show large unexplained differences from the existing network theories. It is therefore of interest to examine in detail the effect of the approximations made in calculating the scattering function, and to introduce an explicit treatment of polydispersity into the calculation. 
The network for which it is simplest to obtain the scattering function is the monodisperse end-linked network considered by Pearson [25]. All subchains have an equal length and certain labelled subchains are distributed randomly within the network in such a way that there will be negligible interference between scattered particles from different labelled subchains. It is more usual in experimental systems to have many crosslinks on a labelled chain. Ullmann [26] considered scattering from a network containing long labelled chains with many crosslinks, assumed to be equally spaced along the chain. $\mathrm{He}$ obtained the scattering function assuming the junctions to be fixed and to deform affinely: the monodisperse junction affine model (M.J.A.). A solution for the monodisperse phantom network (M.P.N.) case (allowing free junction fluctuations) was also obtained by Ullmann, but this was only approximate. In section 7 we derive the exact M.P.N. solution.

As discussed above, if the network is made by forming crosslinks at random sites along the chains in a melt, then the subchain lengths will have a Poisson distribution. This is generally held to correspond to the situation in networks formed by irradiative crosslinking. Warner and Edwards [27] used the replica method to obtain an approximate solution in this case. In this treatment the random positioning of the crosslinks is incorporated by assuming a mean localization of the labelled chain by its crosslinks which is the same at all points. This work has been extended recently by Vilgis and Boue [28] to include the partially restricted crosslink points of the FloryErman model [29] and the slipink treatment of entanglements. In section 8 we give a simple argument which yields the same form for the scattering function as the replica method, and which sheds light on the approximations used in the replica calculation.

The work of Bastide, Herz and Boue [30] has demonstrated that both the monodisperse and the replica methods are in substantial disagreement with the experimental measurements. There are two major differences between the data and the existing theories apparent when looking at the Kratky plot (i.e. $q^{2} S(\mathbf{q})$ against $\mathbf{q}$ ) for $\mathbf{q}$ perpendicular to the stretch direction. The peak at intermediate $\mathbf{q}$ is much lower and broader than predicted by theory, and the convergence of the curve to its limiting value for large $\mathbf{q}$ is much slower than predicted by theory.

In this paper we demonstrate that accounting accurately for the polydispersity of the subchains leads to substantial changes in the predicted shape of the scattering function. In section 9 we use the resistor networks discussed above to obtain the scattering function for a polydisperse junction affine (P.J.A.) network with arbitrary $f(S)$, and apply it to the Poisson distribution case. The polydisperse phan- tom network (P.P.N.) calculation presents considerable difficulty, however we do obtain an approximate solution.

Sections 7-9 are largely mathematical. The reader not interested in the details of the derivations may turn to section 10 , where the conclusions relevant to fitting the experimental data are discussed.

\section{Monodisperse models.}

The intensity of coherent scattering at scattering vector $\mathbf{k}$ is given by

$$
S(\mathbf{k}) \propto \sum_{p q}\left\langle\exp \left(i \mathbf{k} \cdot \mathbf{r}_{p q}\right)\right\rangle
$$

Where $\mathbf{r}_{p q}$ is the vector linking monomers $p$ and $q$ on the labelled subchain (or subchains). Writing this in an integral representation

$$
S(\mathbf{k}) \propto \int_{0}^{s_{0}} \int_{0}^{S_{0}} \mathrm{~d} s \mathrm{~d} s^{\prime}\left\langle\exp \left(i \mathbf{k} \cdot\left(\mathbf{r}(s)-\mathbf{r}\left(s^{\prime}\right)\right)\right)\right\rangle .
$$

The variables $s$ and $s^{\prime}$ represent arc length along the chain of length $S_{0}$. Because of the Gaussian nature of the chain segment $\mathbf{r}(s)-\mathbf{r}\left(s^{\prime}\right)$ we may write

$$
\begin{aligned}
S(\mathbf{k}) \propto & \int_{0}^{S_{0}} \int_{0}^{S_{0}} \mathrm{~d} s \mathrm{~d} s^{\prime} \times \\
& \times \exp \left\{-\frac{1}{2} \sum_{i} k_{i}^{2}\left\langle\left(r_{i}(s)-r_{i}\left(s^{\prime}\right)\right)^{2}\right\rangle\right\} .
\end{aligned}
$$

The sum is over Cartesian components. We will sketch the derivation of the monodisperse models since we will use them in generalizing to the polydisperse systems. For Pearson's end-linked chains :

$$
\begin{aligned}
& r_{i}(s)-r_{i}\left(s^{\prime}\right)= \\
& \quad=\lambda_{i}\left(R_{i}(s)-R_{i}\left(s^{\prime}\right)\right)+\left(\rho_{i}(s)-\rho_{i}\left(s^{\prime}\right)\right) \\
& \left\langle\left(r_{i}(s)-r_{i}\left(s^{\prime}\right)\right)^{2}\right\rangle=\lambda_{i}^{2} \frac{\left|s-s^{\prime}\right|^{2}}{S_{0}^{2}} \times \\
& \quad \times(1-2 / \phi) S_{0}+\left\langle\left(\rho_{i}(s)-\rho_{i}\left(s^{\prime}\right)\right)^{2}\right\rangle .
\end{aligned}
$$

The fluctuations are independent of $\lambda$ therefore when $\lambda=1$ :

$$
\begin{aligned}
\left\langle\left(\rho_{i}(s)\right.\right. & \left.\left.-\rho_{i}\left(s^{\prime}\right)\right)^{2}\right\rangle= \\
& =\left|s-s^{\prime}\right|-\frac{\left|s-s^{\prime}\right|^{2}}{S_{0}^{2}}(1-2 / \phi) S_{0} .
\end{aligned}
$$

Here we have used the «Flory assumption» (assumption 3 of section 1 ). Now defining the dimensionless scattering vector $\mathbf{q}$ by :

$$
q_{i}^{2}=S_{0} k_{i}^{2}
$$

and letting $\left|t-t^{\prime}\right|=\left|s-s^{\prime}\right| / S_{0}$ we obtain : 


$$
\begin{aligned}
S_{\text {self }}(\mathbf{q}) & =S_{0}^{2} \int_{0}^{1} \int_{0}^{1} \mathrm{~d} t \mathrm{~d} t^{\prime} \exp \left\{-\frac{1}{2} \sum_{i} q_{i}^{2}\left(\left|t-t^{\prime}\right|+\left(\lambda_{i}^{2}-1\right)\left|t-t^{\prime}\right|^{2}(1-2 / \phi)\right)\right\} \\
& =2 S_{0}^{2} \int_{0}^{1} \mathrm{~d} t(1-t) \exp \left\{-\frac{1}{2} \sum_{i} q_{i}^{2}\left(t+\left(\lambda_{i}^{2}-1\right) t^{2}(1-2 / \phi)\right)\right\} .
\end{aligned}
$$

The M.J.A. model has $N_{s}$ subchains of length $S_{0}$ forming one long labelled chain crosslinked to many unlabelled chains. Ullmann considers the case of dangling chain ends in detail, and thus distinguishes five different positionings of the arc length variables $s$ and $s^{\prime}$. We follow Bastide, Herz and Boue in assuming that $N_{s}$ is large and dangling ends have a negligible effect. There are then only two cases : $s$ and $s^{\prime}$ on the same subchain or on different subchains.

In the former case $S(\mathbf{q})=S_{\text {self }}(\mathbf{q})$ with $\phi=\infty$. In the latter case let there be $n$ subchains between the two subchains on which $s$ and $s^{\prime}$ are situated $\left(0 \leqslant n \leqslant N_{s}-2\right)$. Since the junction points are assumed fixed the vector $\mathbf{r}(s)-\mathbf{r}\left(s^{\prime}\right)$ is the sum of three independent parts. Introducing the notation :

$$
\Delta_{i}\left(t, t^{\prime}\right) \equiv \frac{\left\langle\left(r_{i}(s)-r_{i}\left(s^{\prime}\right)\right)^{2}\right\rangle}{S_{0}}
$$

We find in this case :

$$
\begin{aligned}
\Delta_{i}\left(t, t^{\prime}\right)=t+\left(\lambda_{i}^{2}-1\right) & t^{2}+ \\
& +\lambda_{i}^{2} n+t^{\prime}+\left(\lambda_{i}^{2}-1\right) t^{\prime 2} .
\end{aligned}
$$

Let

$$
\begin{aligned}
I_{1}(\mathbf{q}) & =\int_{0}^{S_{0}} \mathrm{~d} s \exp \left\{-\frac{1}{2} \sum_{i} k_{i}^{2}\left(s+\left(\lambda_{i}^{2}-1\right) \frac{s^{2}}{S_{0}}\right)\right\} \\
& =S_{0} \int_{0}^{1} \mathrm{~d} t \exp \left\{-\frac{1}{2} \sum_{i} q_{i}^{2}\left(t+\left(\lambda_{i}^{2}-1\right) t^{2}\right)\right\}
\end{aligned}
$$

There are $\left(N_{s}-n-1\right)$ pairs of subchains with $n$ intermediates and each of these pairs must be counted twice to give the correct weighting in comparison to $S_{\text {self }}$, therefore the contribution from subchains with $n$ intermediates is :

$S_{n}(\mathbf{q})=2\left(N_{s}-n-1\right) \exp \left(-\frac{n}{2} \sum_{i} q_{i}^{2} \lambda_{i}^{2}\right) I_{1}(\mathbf{q})^{2}$

Assembling the contributions and normalizing so that the limit of $S(\mathbf{q})$ as $|\mathbf{q}| \rightarrow \infty$ is $1 / q^{2}$ we find

$$
S_{\mathrm{MJA}}(\mathbf{q})=\frac{1}{4 N_{s} S_{0}^{2}}\left(N_{s} S_{\mathrm{self}}(\mathbf{q})+2 I_{1}(\mathbf{q})^{2} \sum_{n=0}^{N_{s}-2}\left(N_{s}-n-1\right) \exp \left(-\frac{n}{2} \sum_{i} q_{i}^{2} \lambda_{i}^{2}\right)\right)
$$

It is possible to express $S_{\text {self }}(\mathbf{q})$ and $I_{1}(\mathbf{q})$ in terms of error functions and Dawsons integral but we have evaluated the integrals numerically.

Ullmann uses the equivalent equation to (7.7) for finite $\phi$. This is only an approximation since the three parts are no longer independent. The problem can be viewed in terms of the equivalent circuit of figure 9i. The resistor $Y$ represents a tree with $(\phi-2)$ branches in the top layer rather than $(\phi-1)$ as is the case for $X$. We desire to calculate the resistance between points 1 and 2, which is the mean square of the fluctuations between the points. We use the notation $\rho_{1}=$ any one component of the fluctuation of point 1 from its mean position, and $\rho_{12}=$ any one component of the fluctuation in the separation of points 1 and 2 .

$$
S_{12}^{\mathrm{cff}}=\left\langle\rho_{12}^{2}\right\rangle=\left\langle\rho_{1}^{2}\right\rangle+\left\langle\rho_{2}^{2}\right\rangle-2\left\langle\rho_{1} \rho_{2}\right\rangle
$$

$\left\langle\rho_{1}^{2}\right\rangle$ is the resistance to earth via all routes from point 1 (Fig. 9ii).

$$
\begin{aligned}
\left\langle\rho_{1}^{2}\right\rangle & =\left(\frac{1}{S_{0} t+X}+\frac{1}{S_{0}(1-t)+X}\right)^{-1} \\
& =S_{0} \frac{(\phi-1)}{\phi(\phi-2)}\left(1+t(1-t) \frac{(\phi-2)^{2}}{(\phi-1)}\right)
\end{aligned}
$$

and similarly for $\left\langle\rho_{2}^{2}\right\rangle$. To find $\left\langle\rho_{1} \rho_{2}\right\rangle$ we use Ullman's argument that :

$$
\rho_{\nu}=\frac{\rho_{\mu}}{(\phi-1)^{n}}+\varepsilon
$$

where the first term is the equilibrium displacement of junction $\nu$ caused by the motion of junction $\mu$, and $\varepsilon$ is a displacement caused by the instantaneous 


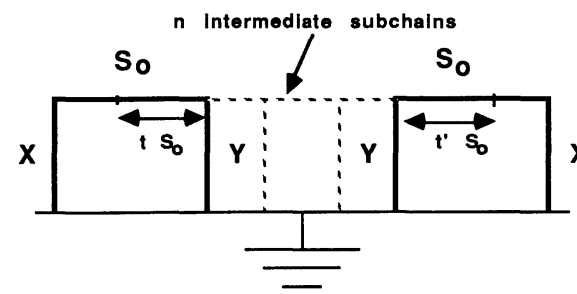

(i)

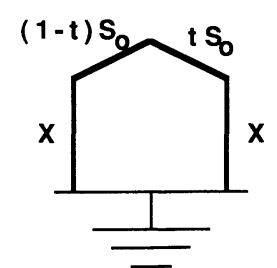

(ii)
Fig. 9. - i) Circuit for the Monodisperse Phantom Network model ; ii) resistance to ground from an arbitrary point.

disequilibrium of forces, and is uncorrelated with $\rho_{\mu}$ or $\rho_{\nu}$. Hence :

$$
\left\langle\rho_{\mu} \rho_{\nu}\right\rangle=\frac{\left\langle\rho_{\mu}^{2}\right\rangle}{(\phi-1)^{n}} .
$$

We now show that the correlation in the motions of the arbitrary points 1 and 2 may be expressed entirely in terms of the correlation in the motions of the junction points. The equilibrium displacements of points 1 and 2 are linear interpolations of the displacements of the junctions either side of them.

$$
\left.\begin{array}{ll} 
& \rho_{1}=t \rho_{\mu-1}+(1-t) \rho_{\mu}+\varepsilon \\
\text { and } & \\
& \rho_{2}=t^{\prime} \rho_{\nu+1}+\left(1-t^{\prime}\right) \rho_{\nu}+\varepsilon^{\prime}
\end{array}\right\}
$$

$$
\begin{aligned}
\therefore & \left\langle\rho_{1} \rho_{2}\right\rangle=t t^{\prime}\left\langle\rho_{\mu-1} \rho_{\nu+1}\right\rangle+ \\
& +t\left(1-t^{\prime}\right)\left\langle\rho_{\mu-1} \rho_{\nu}\right\rangle+(1-t) t^{\prime}\left\langle\rho_{\mu} \rho_{\nu+1}\right\rangle+ \\
& +(1-t)\left(1-t^{\prime}\right)\left\langle\rho_{\mu} \rho_{\nu}\right\rangle .
\end{aligned}
$$

This is an important result since it does not depend on the network being monodisperse. However it is only in the monodisperse case that we can evaluate the expression using (7.13). Combining $(7.11,7.12,7.13,7.15)$ yields an expression for $\left\langle\rho_{12}^{2}\right\rangle$. Before deformation :

$$
\Delta_{i}\left(t, t^{\prime}\right)=n+t+t^{\prime}
$$

and therefore after deformation :

$$
\begin{aligned}
\Delta_{i}\left(t, t^{\prime}\right)=\lambda_{i}^{2}\left(n+t+t^{\prime}\right) & + \\
+ & \left(1-\lambda_{i}^{2}\right)\left\langle\rho_{12}^{2}\right\rangle / S_{0}
\end{aligned}
$$

which may be substituted into the exponential in the scattering function integral. This is much more difficult to evaluate than the M.J.A. case since the variables $t$ and $t^{\prime}$ are no longer separable so we have a two-dimensional integral instead of the square of a one-dimensional integral. Also the $n$ factor is not separable either so we must do a different integral for each value of $n$ in the sum. We have not evaluated the M.P.N. solution. In the light of the results below we believe it would not differ substantially from the other models which we discuss.

\section{The replica result.}

The result of Warner and Edwards [27] may be written

$$
\begin{gathered}
S_{\text {rep }}(\mathbf{k}) \propto \int_{0}^{N_{s} s_{0}} \int_{0}^{N_{s} s_{0}} \mathrm{~d} s \mathrm{~d} s^{\prime} \exp \left\{-\frac{1}{2} \sum_{i} k_{i}^{2}\left(\lambda_{i}^{2}\left|s-s^{\prime}\right|+\left(1-\lambda_{i}^{2}\right) S_{0} C\left[1-\exp \left(-\frac{\left|s-s^{\prime}\right|}{S_{0} C}\right)\right]\right)\right\} \\
S_{\text {rep }}(\mathbf{q})=\frac{1}{2 N_{s}} \int_{0}^{N_{s}} \mathrm{~d} t\left(N_{s}-t\right) \exp \left\{-\frac{1}{2} \sum_{i} q_{i}^{2}\left[\lambda_{i}^{2} t+\left(1-\lambda_{i}^{2}\right) C\left(1-\mathrm{e}^{-t / C}\right)\right]\right\}
\end{gathered}
$$

The normalization is as before, and again $t$ is arc length measured in units of $S_{0}$. The constant $w=6 N_{x} / N \ell^{2}$ of references $[6,27]$ has been replaced by $C$ in our notation, where

$$
C=1 / S_{0} w
$$

Now with $N_{0}=$ the mean number of monomers on a subchain and $N=N_{0} N_{s}=$ the total number of monomers on a chain, we have

$$
C=\frac{3}{N_{0} \ell^{2}} \frac{N \ell^{2}}{6 N_{x}}=\frac{1}{2} \frac{N_{s}}{N_{x}}=1 \text { for } \phi=4 .
$$

This result was generalized by one of us [31] to arbitrary even functionality, in which case the same functional form as (8.1) was found with

$$
\left.\begin{array}{l}
w=\frac{6 N_{x}}{N I^{2}}\left(\frac{\phi}{2}-1\right) \\
C=\frac{1}{2} \frac{\phi}{(\phi-2)} \cdot
\end{array}\right\}
$$

It was then suggested that analytic continuation of this result to odd functionality was valid.

The replica treatment of Gaussian networks is based on the idea that topological constraints divide 
the configuration set of the network into many distinct sets not accessible to each other without breaking and reforming bonds. The method deals elegantly with this problem, but unfortunately evaluation of the free energy and the scattering function relies on the introduction of an approximate Green function in which the chain is localized by its crosslinks in a harmonic potential well. The depth of this well is determined by a variational principle which minimizes the free energy for the value of $w$ in (8.4). All information on positioning of the crosslinks is lost. All points on the chain become equivalent. We are thus led to consider the alternative mean field representation below.

The labelled chain is, in the resistance analogy, a wire of unit resistivity connected to earth by a continuous medium of conductivity $\sigma$ (Fig. 10i). An element $\mathrm{d} t$ of the chain is represented in figure $10 \mathrm{ii}$ from which we have :

$$
I=-\frac{\partial V}{\partial t} \quad V=\frac{1}{\sigma} \frac{\partial I}{\partial t}
$$

writing $\sigma=1 / C^{2}$ (anticipating the result (8.9)) we find :

$$
\frac{\partial^{2} V}{\partial t^{2}}=-\frac{1}{C^{2}} V(t)
$$

Now put a current $I_{\text {in }}$ into the wire at $t=0$, which maintains the voltage $V(0)=V_{0}$. The solution for $V(t)$ is :

$$
\begin{array}{r}
V(t)=V^{+}=V_{0} \exp (-t / C) \quad t>0 ; \\
V^{-}=V_{0} \exp (+t / C) \quad t<0 . \\
I_{\text {in }}=\left[-\frac{\partial V^{+}}{\partial t}+\frac{\partial V^{-}}{\partial t}\right]_{t=0}=\frac{2 V_{0}}{C} .
\end{array}
$$

Hence the resistance to ground from any point is

$$
\left\langle\rho_{1}^{2}\right\rangle / S_{0}=C / 2 \text {. }
$$

In a similar way, putting current $I_{\text {in }}$ into the wire at the origin and taking the same current out at another point $t$ we find that the resistance between two points distance $t$ apart is :

$$
S_{12}^{\text {eff }} / S_{0}=C(1-\exp (-t / C)) .
$$

This expression tends to twice the resistance to ground in the limit $t \rightarrow \infty$ where the two points become independent.

The Flory assumption applied to this model means that before deformation :

$$
\Delta_{i}(t, 0)=t
$$

and therefore after deformation

$$
\Delta_{i}(t, 0)=\lambda_{i}^{2} t+\left(1-\lambda_{i}^{2}\right) C(1-\exp (-t / C))
$$

which is exactly the factor occurring in the replica form of the scattering function (8.1) i.e. the above model deforms in a way which gives rise directly to $S_{\text {rep }}(\mathbf{q})$. For clarity we will continue to refer to (8.1) as the replica formula for the scattering function, though we will view it in the light of the above model rather than its original derivation.

We must now address the issue of the value of the constant $C$. The resistance to ground in the smeared out model is $C / 2$, therefore we choose $C$ to be twice the average resistance to ground in the real network (measured in units of $S_{0}$ ). For a monodisperse network we may use (7.12) :

$$
\begin{aligned}
C_{\text {mono }} & =\int_{0}^{1} \mathrm{~d} t \frac{2(\phi-1)}{\phi(\phi-2)}\left(1+t(1-t) \frac{(\phi-2)^{2}}{(\phi-1)}\right) \\
& =\frac{2(\phi-1)}{\phi(\phi-2)}\left(1+\frac{(\phi-2)^{2}}{6(\phi-1)}\right) .
\end{aligned}
$$

In the polydisperse case we have :

$$
\begin{gathered}
\frac{1}{\left\langle\rho_{1}^{2}\right\rangle}=\frac{1}{s+X_{1}}+\frac{1}{S-s+X_{2}} \\
C=\frac{2\left\langle\rho_{1}^{2}\right\rangle}{S_{0}}=\frac{2}{S_{0}}\left\langle\frac{\left(s+X_{1}\right)\left(S-s+X_{2}\right)}{S+X_{1}+X_{2}}\right\rangle_{\text {average over monomers }} \\
=\frac{2}{S_{0}}\left\langle\frac{1}{S_{0}} \int_{0}^{s} \mathrm{~d} s \frac{\left(s+X_{1}\right)\left(S-s+X_{2}\right)}{S+X_{1}+X_{2}}\right\rangle_{s, X_{1}, X_{2}} \\
=\frac{1}{S_{0}^{2}}\left\langle\left.\frac{\frac{1}{3} S^{3}+S^{2}\left(X_{1}+X_{2}\right)+2 S X_{1} X_{2}}{S+X_{1}+X_{2}}\right|_{s, X_{1}, X_{2}} .\right.
\end{gathered}
$$




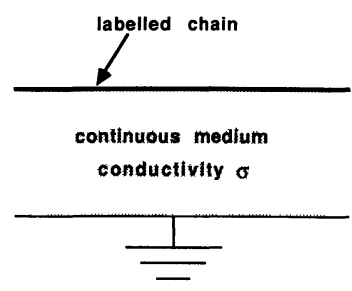

(i)

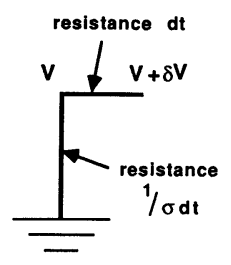

(ii)
Fig. 10. - Mean field model with smeared out resistance to ground. This is a realization of the effects of the approximations made in the replica calculation, and yields the same answer for the scattering function.

We are interested in this quantity for the Poisson distribution of subchain lengths. For $\phi=3$ evaluating the expression with the calculated $g(X)$ gives $C=1.53 \pm 0.01$, which is remarkably close to the value $C=C_{\text {rep }}=3 / 2$ obtained by the replica calculation of (8.4) extended to $\phi=3$. Table IV compares $C_{\text {rep }}$ and $C_{\text {mono }}$ to the best estimate of $C$ from the current data for iseveral values of $\phi$. For $\phi \neq 3$ the best estimate is calculated as a midpoint between upper and lower bounds. The lower bound is the mean field solution obtained by putting $X_{1}=X_{2}=X_{\mathrm{M}}$ in (8.12) and only integrating over $S$. The upper bound is obtained by putting $X_{1}=$ $X_{2}=X_{0}$ (which is itself estimated from (B.8)) and integrating over $S$. For $\phi \geqslant 6$ these bounds are very close. The limit $\phi \rightarrow \infty$ of (8.12) is :

$$
C=1 / S_{0}^{2} \cdot\left\langle S^{2} / 3\right\rangle=2 / 3
$$

which is different from the limit of $C_{\text {mono }}$ and $C_{\text {rep }}$. In short it is found that $C_{\text {rep }}$ is an accurate approximation for $\phi=3$ and $\phi=4$ but that it has the wrong limit as $\phi \rightarrow \infty$, and that the mean field result (lower bound) is an accurate approximation for $\phi>4$.

Figure 11 shows the replica scattering functions for several values of $C$ compared to the junction affine models. The functions are evaluated for a sample under uniaxial extension $(\lambda=4.6)$ with $\mathbf{q}$ perpendicular to the stretching direction and $N_{s}=50$. We choose these values for comparison to reference [33]. The curves are discussed in section 10.

\section{Polydisperse models.}

For the polydisperse junction affine model we again distinguish between the cases of $s$ and $s^{\prime}$ on the same
Table IV. - Various approximations for the constant $C$ in the replica formula for the scattering function.

$\begin{array}{cccc}\phi & C_{\text {mono }} & C_{\text {rep }} & C \text { (best estimate) } \\ 3 & 13 / 9 & 3 / 2 & 1.53 \pm 0.01 \\ 4 & 11 / 12 & 1 & \sim 1.0 \\ 6 & 23 / 36 & 3 / 4 & \sim 0.8 \\ 10 & 59 / 120 & 5 / 8 & \sim 0.72 \\ \infty & 1 / 3 & 1 / 2 & 2 / 3\end{array}$

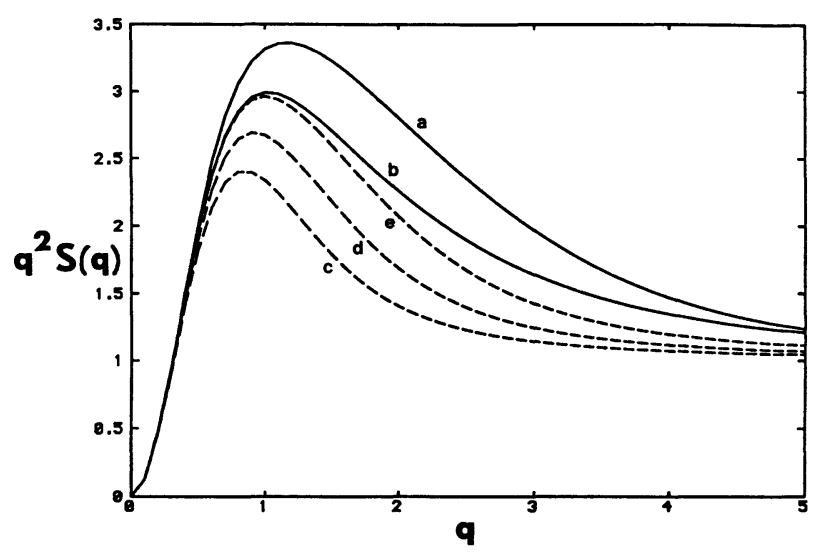

Fig. 11. - Kratky plots $\left(q^{2} S(q)\right)$ for perpendicular scattering from a uniaxially extended network with $\lambda=4.6$ and the number of subchains (meshes) along the labelled chain $N_{s}=50$. a) Monodisperse Junction Affine ; b) Polydisperse Junction Affine (c) (d) and (e) Replica model for $\phi=3(C=3 / 2), \phi=4(C=1)$, and $\phi=\infty$ $(C=2 / 3)$. The difference between (a) and (b) is attributable entirely to polydispersity. The replica curves, which model the effects of junction point fluctuations, converge much more rapidly than the junction affine curves. The dimensionless wave vector $q$ is scaled relative to the r.m.s. mesh size (see Eq. (7.5)).

subchain and on different subchains. In the former case (7.6) can be generalized as

$$
\begin{aligned}
S_{\text {self }}(\mathbf{q})=2 S_{0}^{2} & T^{2} \int_{0}^{1} \mathrm{~d} t(1-t) \times \\
& \times \exp \left\{-\frac{1}{2} \sum_{i} q_{i}^{2} T\left(t+\left(\lambda_{i}^{2}-1\right) t^{2}\right)\right\}
\end{aligned}
$$

where $T=S / S_{0}$ and $t=s / S$.

This must be averaged over the distribution of subchain lengths. In the Poisson network $f(T)=\mathrm{e}^{-T}$ :

$$
G_{\text {self }}(\mathbf{q})=\int_{0}^{\infty} \mathrm{e}^{-T} S_{\text {self }}(\mathbf{q}) \mathrm{d} t=4 S_{0}^{2} \int_{0}^{1} \mathrm{~d} t \frac{(1-t)}{\left(1+\frac{1}{2} \sum_{i} q_{i}^{2}\left(t+\left(\lambda_{i}^{2}-1\right) t^{2}\right)\right)^{3}} .
$$


In the second case the junction affine assumption allows a generalization of (7.8) :

$$
\begin{aligned}
\Delta_{i}\left(t, t^{\prime}\right)=T( & \left.t+\left(\lambda_{i}^{2}-1\right) t^{2}\right)+ \\
& +\lambda_{i}^{2} T_{n}+T^{\prime}\left(t^{\prime}+\left(\lambda_{i}^{2}-1\right) t^{\prime 2}\right)
\end{aligned}
$$

where $T$ and $T^{\prime}$ are independent lengths and $T_{n}$ is the sum of $n$ independent values of $T$. (7.9) becomes :

$$
\begin{aligned}
I_{1}(\mathbf{q})=S_{0} T \int_{0}^{1} \mathrm{~d} t \times & \\
& \times \exp \left\{-\frac{1}{2} \sum_{i} q_{i}^{2} T\left(t+\left(\lambda_{i}^{2}-1\right) t^{2}\right)\right\}
\end{aligned}
$$

which when averaged over $T$ gives :

$$
G_{1}(\mathbf{q})=S_{0} \int_{0}^{1} \mathrm{~d} t \frac{1}{\left(1+\frac{1}{2} \sum_{i} q_{i}^{2}\left(t+\left(\lambda_{i}^{2}-1\right) t^{2}\right)\right)^{2}}
$$

$T_{n}$ has a probability distribution given by :

$$
\begin{aligned}
f_{n}\left(T_{n}\right) & =\iiint_{0}^{\infty} \ldots \mathrm{d} T \mathrm{~d} T^{\prime} \mathrm{d} T^{\prime \prime} \ldots f(T) \times \\
& \times f\left(T^{\prime}\right) f\left(T^{\prime \prime}\right) \ldots \delta\left(T_{n}-T-T^{\prime}-T^{\prime \prime}-\ldots\right)
\end{aligned}
$$

which can be evaluated using the convolution theorem for Laplace transforms. For $f(T)=\mathrm{e}^{-T}$ we have :

$$
f_{n}\left(T_{n}\right)=\frac{\left(T_{n}\right)^{n-1}}{(n-1) !} \mathrm{e}^{-T_{n}}
$$

The factor in (9.2) involving $T_{n}$ is now averaged over this distribution.

$$
\int_{0}^{\infty} \mathrm{d} T_{n} \frac{\left(T_{n}\right)^{n-1}}{(n-1) !} \exp \left(-T_{n}\right) \exp \left(-\frac{1}{2} T_{n} \sum_{i} q_{i}^{2} \lambda_{i}^{2}\right)=\frac{1}{\left(1+\frac{1}{2} \sum_{i} \lambda_{i}^{2} q_{i}^{2}\right)^{n}}
$$

hence

$$
S_{\mathrm{PJA}}(\mathbf{q})=\frac{1}{4 N_{s} S_{0}^{2}}\left(N_{s} G_{\text {self }}(\mathbf{q})+2 G_{1}(\mathbf{q})^{2} \sum_{n=0}^{N_{s}-2}\left(N_{s}-n-1\right) \frac{1}{\left(1+\frac{1}{2} \sum_{i} \lambda_{i}^{2} q_{i}^{2}\right)^{n}}\right)
$$

This has been evaluated for $N_{s}=50$ and $\lambda=4.6$ in figure 11 .

A principal result from experiment is that the peak in the Kratky plot for perpendicular scattering is much lower than predicted by the monodisperse models. We note that the P.J.A. curve is significantly lower than the M.J.A. Also, the deformation in a phantom network model will always be less than in a junction affine model for the same $\lambda$. Therefore the perpendicular scattering curve will be lower (closer to the curve for the undeformed sample) for phantom network models. It is therefore of interest to attempt a proper treatment of the polydisperse phantom network.

As was noted in the discussion of the M.P.N., when the junctions are allowed to fluctuate the term in $\Delta_{i}\left(t, t^{\prime}\right)$ involving the number of intermediate subchains $n$ does not separate conveniently from the other variables and it is necessary to do a separate integral for each $n$ and sum these. From figure 9 it can be seen that the number of variables involved is $(n+2) S$-values, $2 X$-values, $(n+1) Y$-values plus $t$ and $t^{\prime}$. Performing the integration over all these variables is a major computational task. We therefore make the simplification of replacing all the $X$ variables by $X_{\mathrm{M}}$ and all the $Y$ variables by $Y_{\mathrm{M}}$. As stated above, $Y$ is the resistance of a tree with $\phi-2$ branches on the top layer instead of $\phi-1$. Hence $Y_{M}$ is defined by

$$
\frac{1}{Y_{M}}=(\phi-2) \int_{0}^{\infty} \mathrm{d} T \frac{\mathrm{e}^{-T}}{T+X_{\mathrm{M}}}=\frac{(\phi-2)}{(\phi-1)} \frac{1}{X_{\mathrm{M}}} \text {. }
$$

We are interested in $\phi=4$ where $X_{M}=0.247$ and $Y_{\mathrm{M}}=0.371$ (in units of $S_{0}$ ). Because of the relatively narrow distribution of $X$ it is not unreasonable to replace $X$ by a single value. The major effect of polydispersity lies in the variation of the $S$ variables (those in the labelled chain itself). It was also noted above that the mode of the $g(X)$ curve $\approx X_{\mathrm{M}}$, and therefore we prefer to set $X=X_{M}$ rather than $X=X_{0}$. 


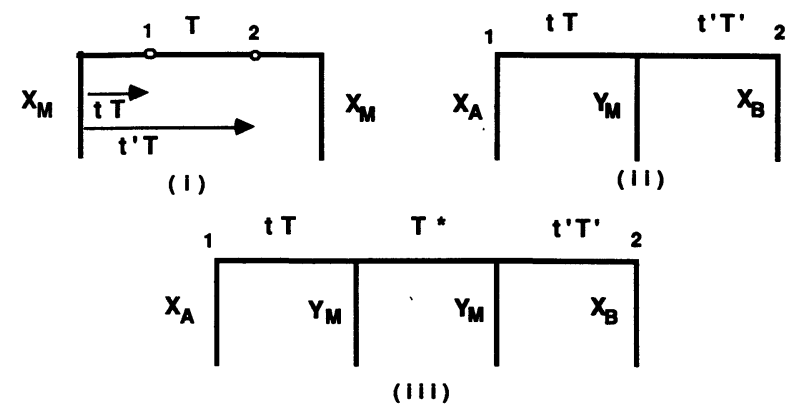

Fig. 12. - The three simplest terms contributing to the Polydisperse Phantom Network scattering function. (Cf. Fig. 19 and analysis in Sect. 9.)

The three simplest contributions to the scattering function are shown in figure 12 .

i) $t$ and $t^{\prime}$ on the same subchain :

$$
\frac{S_{12}^{\text {eff }}}{S_{0}}=T\left|t-t^{\prime}\right|-\frac{T^{2}\left|t-t^{\prime}\right|^{2}}{Z_{\mathrm{M}}+T}
$$

which leads in the usual way to :

$$
\begin{gathered}
\Delta_{i}\left(t, t^{\prime}\right)=T\left(\left|t-t^{\prime}\right|+\left(\lambda^{2}-1\right) \frac{\left|t-t^{\prime}\right|^{2}}{\left(1+\frac{Z_{\mathrm{M}}}{T}\right)}\right) \\
\begin{aligned}
G_{\text {self }}(\mathbf{q})=\int_{0}^{\infty} \mathrm{d} T 2 S_{0}^{2} T^{2} \mathrm{e}^{-T} \int_{0}^{1} \mathrm{~d} t(1-t) \times \\
\quad \times \exp \left(-\frac{1}{2} \sum_{i} q_{i}^{2} \Delta_{i}(t, 0)\right)
\end{aligned}
\end{gathered}
$$

ii) $t$ and $t^{\prime}$ on neighbouring subchains $(n=0)$ :

We require the resistance between points 1 and 2 in figure 12ii (which are not necessarily junction points) :

$$
X_{\mathrm{A}}=X_{\mathrm{M}}+T(1-t) \quad X_{\mathrm{B}}=X_{\mathrm{M}}+T^{\prime}\left(1-t^{\prime}\right)
$$

with $T$ and $T^{\prime}$ two independent subchain lengths. Solving Kirchhoff's equations yields :

$$
\begin{gathered}
\frac{S_{12}^{\text {eff }}}{S_{0}}=\frac{X_{\mathrm{A}} X_{\mathrm{B}}\left(T t+T^{\prime} t^{\prime}\right)+T t T^{\prime} t^{\prime}\left(X_{\mathrm{A}}+X_{\mathrm{B}}\right)+Y_{\mathrm{M}}\left(T t+T^{\prime} t^{\prime}\right)\left(X_{\mathrm{A}}+X_{\mathrm{B}}\right)}{\left(X_{\mathrm{M}}+T+Y_{\mathrm{M}}\right)\left(X_{\mathrm{M}}+T+Y_{\mathrm{M}}\right)-Y_{\mathrm{M}}^{2}} \\
\Delta_{i}\left(t, t^{\prime}\right)=\lambda_{i}^{2}\left(T t+T^{\prime} t^{\prime}\right)+\left(1-\lambda_{i}^{2}\right) \frac{S_{12}^{\text {eff }}}{S_{0}} \\
G_{n=0}(\mathbf{q})=\iint_{0}^{\infty} \mathrm{d} T \mathrm{~d} T^{\prime} S_{0}^{2} T T^{\prime} \mathrm{e}^{-T-T^{\prime}} \iint_{0}^{1} \mathrm{~d} t \mathrm{~d} t^{\prime} \exp \left(-\frac{1}{2} \sum_{i} q_{i}^{2} \Delta_{i}\left(t, t^{\prime}\right)\right)
\end{gathered}
$$

iii) the $n=1$ contribution :

The notation is as above with the addition of a third independent subchain $S^{*}=S_{0} T^{*}$. We find :

$$
\begin{gathered}
X_{\mathrm{B}}\left[T^{*}+\gamma t T T^{*}+t T+t^{\prime} T^{\prime}\right]\left\{\left[\left(1+\gamma T^{*}\right)\left(T^{\prime}+X_{\mathrm{M}}\right)+T^{*}\right]-\left(T+X_{\mathrm{M}}\right)\right\} \\
\frac{S_{12}^{\mathrm{eff}}}{S_{0}}=\frac{X_{\mathrm{A}}\left[T^{*}+\gamma t^{\prime} T^{\prime} T^{*}+t T+t^{\prime} T^{\prime}\right]\left\{\left[\left(1+\gamma T^{*}\right)\left(T+X_{\mathrm{M}}\right)+T^{*}\right]-\left(T^{\prime}+X_{\mathrm{M}}\right)\right\}}{\left[\left(1+\gamma T^{*}\right)\left(T+X_{\mathrm{M}}\right)+T^{*}\right]\left[\left(1+\gamma T^{*}\right)\left(T^{\prime}+X_{\mathrm{M}}\right)+T^{*}\right]-\left(T+X_{\mathrm{M}}\right)\left(T^{\prime}+X_{\mathrm{M}}\right)}
\end{gathered}
$$

where $\gamma=1 / Y_{\mathrm{M}}$

$$
\begin{gathered}
\Delta_{i}\left(t, t^{\prime}\right)=\lambda_{i}^{2}\left(T t+T^{\prime} t^{\prime}+T^{*}\right)+\left(1-\lambda_{i}^{2}\right) \frac{S_{12}^{\text {eff }}}{S_{0}} \\
G_{n=1}(\mathbf{q})=\iiint_{0}^{\infty} \mathrm{d} T \mathrm{~d} T^{\prime} \mathrm{d} T^{*} S_{0}^{2} T T^{\prime} \mathrm{e}^{-T-T^{\prime}-T^{*}} \iint_{0}^{1} \mathrm{~d} t \mathrm{~d} t^{\prime} \exp \left(-\frac{1}{2} \sum_{i} q_{i}^{2} \Delta_{i}\left(t, t^{\prime}\right)\right) .
\end{gathered}
$$

For $n>1$ even the initial step of solving Kirchhoff's equations is not easy and it is clear that some further approximation is necessary. We might hope that the contributions from $n>1$ would be very small since $\Delta_{i}\left(t, t^{\prime}\right)$ appearing in the exponential is large in this case. If this were true then the final scattering function would not be sensitive to the approximation used, however crude this might be. Unfortunately this proves not to be the case, as is discussed further below and shown by figure 19 . We therefore must attempt some sensible approximation for $n>1$, preferably in which the whole range of $n$ can be treated in one integral.

We will again make use of figure $12 \mathrm{iii}$, but the 
resistor $T^{*}$, instead of representing just one subchain, will represent the resistance of the intermediate chain length regardless of the number of subchains within this length. The replica approximation for the point to point resistance (8.9) may be used :

$$
\text { resistance }=C\left(1-\exp \left(-T^{*} / C\right)\right) \text {. }
$$

This expression is substituted into (9.15) in place of $T^{*}$ every time it occurs. Equation (9.16) remains valid with the new value of $S_{12}^{\text {eff }}$, and (9.17) becomes :

$$
\begin{aligned}
G_{n \geqslant 1}(\mathbf{q})=\iint_{0}^{\infty} \mathrm{d} T \mathrm{~d} T^{\prime} S_{0}^{2} T T^{\prime} \mathrm{e}^{-T-T^{\prime}} \int_{0}^{N_{s}-2} \mathrm{~d} T^{*}\left(N_{s}-2-T^{*}\right) \iint_{0}^{1} \mathrm{~d} t \mathrm{~d} t^{\prime} \times & \\
& \times \exp \left(-\frac{1}{2} \sum_{i} q_{i}^{2} \Delta_{i}\left(t, t^{\prime}\right)\right) .
\end{aligned}
$$

Thus we have an approximation for the contribution to the scattering function from all values of $n \geqslant 1$. Adding cases (i) and (ii) to this we obtain :

$$
\begin{aligned}
S_{\mathrm{PPN}}(\mathbf{q}) & =\frac{1}{4 N_{s} S_{0}^{2}}\left(N_{S} G_{\text {self }}(\mathbf{q})+\right. \\
& \left.+2\left(N_{s}-1\right) G_{n=0}(\mathbf{q})+2 G_{n \geqslant 1}(\mathbf{q})\right) .
\end{aligned}
$$

The Kratky plot for this approximation to the P.P.N. is shown in figure 13. It is seen to be extremely close to the replica curve with $C=1$, suggesting that the replica form was initially a very good approximation to the real answer. There remain, of course, numerous approximations in the P.P.N. form presented here and it is difficult to be sure of their effect. However it is possible to obtain a lower bound on the curve in a simple way, as follows.

In all models $\Delta_{i}(t, 0)$ is of a form similar to (8.10), i.e. :

$$
\Delta_{i}(t, 0)=\lambda_{i}^{2} t+\left(1-\lambda_{i}^{2}\right) \times \ll \text { m.s. fluctuations » }
$$

These mean square fluctuations cannot be greater than $C$ for any value of $t$, since this is the value if the two points are independent. If the fluctuations are set to $C$ for all $t$ this maximizes $\Delta$ in the perpendicular direction (where $\lambda^{2}<1$ ) and therefore minimizes the scattering function. However $\Delta_{i}(t, 0)$ cannot be greater than $t$ : the dimensions must decrease in the perpendicular direction when the material is stretched. Therefore we set :

$$
\begin{aligned}
\Delta_{i}(t, 0) & =t & & t \leqslant C \\
& =\lambda_{i}^{2} t+\left(1-\lambda_{i}^{2}\right) C & & t>C
\end{aligned}
$$

with $C=1$ for $\phi=4$ as above. This situation is of course completely unphysical, yet it provides a useful lower bound to the perpendicular scattering function and an upper bound to the parallel scattering function. (If the special case for $t<C$ were not included then $\Delta$ would become negative in the parallel direction and the scattering function would not converge). The bound for the perpendicular curve is seen in figure 13 to be extremely close to the replica curve and the P.P.N. approximation. In the parallel direction (Fig. 14) the upper bound is not close to the other models and therefore does not provide such a useful guide.

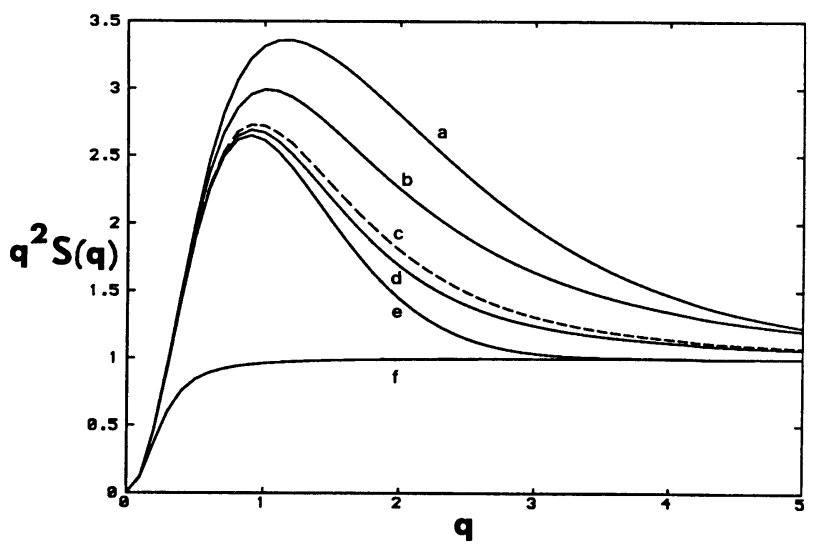

Fig. 13. - Perpendicular scattering from a uniaxially extended network with $\lambda=4.6$ and $N_{\mathrm{s}}=50$. a) M.J.A.; b) P.J.A. ; c) P.P.N. approximation ; d) replica $(C=1)$; e) limiting curve ; f) unstrained network. The proximity of curves (c) and (d) indicates that the replica treatment was initially a good approximation to the P.P.N. Scattering functions for all Gaussian models with this value of $N_{\mathrm{s}}$ must lie above curve (e), this means that it is necessary to assume a substantially smaller $N_{s}$ (longer mesh size) to match the experimental data (see Fig. 15).

\section{Discussion and comparison with experiment.}

Since the real networks may be expected to behave in a way intermediate between the junction affine and the fully fluctuating phantom networks we wish to compare the experimental data to one junction 


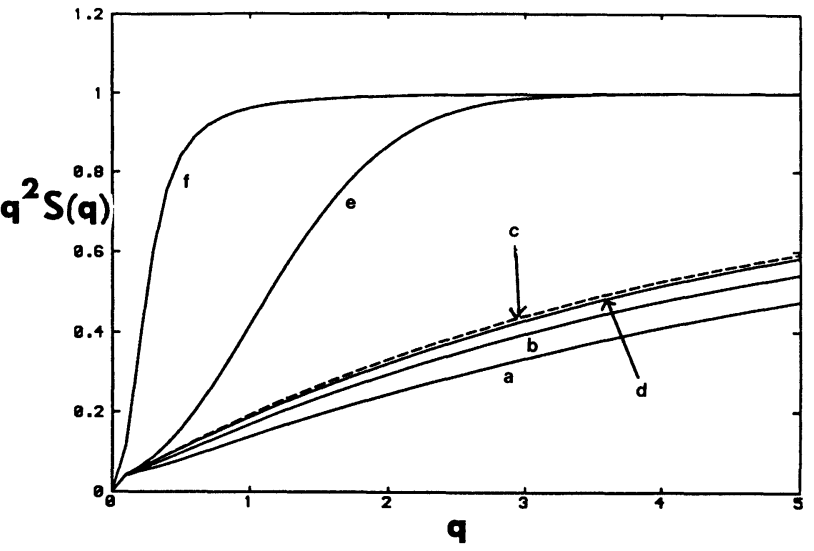

Fig. 14. - As figure 13 for Parallel scattering. The limit curve (e) does not provide a useful upper bound in this case.

affine model and one phantom network model. Figures 13 and 14 demonstrate the main findings of sections 7-9 relevant to our choice of models.

The junction affine models (curves $a$ and b) predict the most anisotropic scattering (strongest perpendicular scattering and weakest parallel scattering). In the perpendicular direction both the Monodisperse and Polydisperse Junction Affine models show slow convergence of the $q^{2} S(q)$ curves to the limiting value of 1 . The polydisperse model derived in section 9 can be seen to differ substantially from the monodisperse theory over the range of $q$ which is of interest. Since polydispersity of subchain lengths is clearly present in the radiatively crosslinked experimental networks we choose the P.J.A. as one of our models for comparison with data.

The replica scattering function (curve c) was originally derived as an approximation to scattering from the Polydisperse Phantom Network. It was shown in section 8 that it corresponds to the extremely simple model of figure 10 which contains no information on positioning of crosslinks, and in which all the unlabelled network chains are represented by a continuous medium. In spite of this apparent crudity it was found to be a very good approximation to the true P.P.N. scattering function. The best estimate for the P.P.N. function derived in section 9 (curve d) differs very little from the replica function. We therefore choose the replica function for comparison to the data in view of its much greater simplicity and ease of evaluation.

We have investigated two sets of experimental data, both made on polystyrene gels crosslinked by irradiation in concentrated solution. The deswollen gel data is taken from Bastide, Herz and Boue (Fig. 4) [30], and is for scattering from labelled paths of two different lengths in a gel deswollen to $1 / 10$ its original volume. The scattering should be equivalent to that in the perpendicular direction from a network in uniaxial extension with $\lambda=4.6$, since $10^{-1 / 3} \approx$ $4.6^{-1 / 2}$. The data for uniaxially extended networks is taken from Bastide and Boue (Fig. 1) [32]. It measures both parallel and perpendicular scattering from networks with $\lambda=1.46$ and 4.6.

For the long path in the deswollen gel it was estimated in the original paper that $\geqslant 50$ crosslinks were present on a typical chain, and that the typical end to end distance of a subchain (or mesh) was $\sim 125 \AA$. The curves in figure 13 are evaluated for $N_{s}=50$. Glancing at the experimental data points in figure 15 it is evident that all the curves have a peak of a much higher intensity than the data. Curve e represents a lower bound for all Gaussian models with this value of $\lambda$ and $N_{s}$. (Derivation of the limiting curve is contained in Sect. 9.) Thus no model based on phantom Gaussian chains will match the data if we retain the assumption of $\geqslant 50$ crosslinks.

In figure 15 we have plotted a series of theoretical curves for the P.J.A. model corresponding to different numbers of subchains on the labelled chain. The mean subchain length $S_{0}$ is held constant, hence the curves correspond to different total chain lengths with the same crosslink density. The $N_{s}$ values chosen are $24,18,12$ and 6 , together with a curve for the unstrained network with $N_{s}=6$. The wave vector scale is measured in dimensionless units $q=k \sqrt{S_{0}}$ (see Sect. 7). We view $S_{0}$ as a scale factor to be determined by sliding the experimental data (in $\AA^{-1}$ ) to best fit into the series of curves.

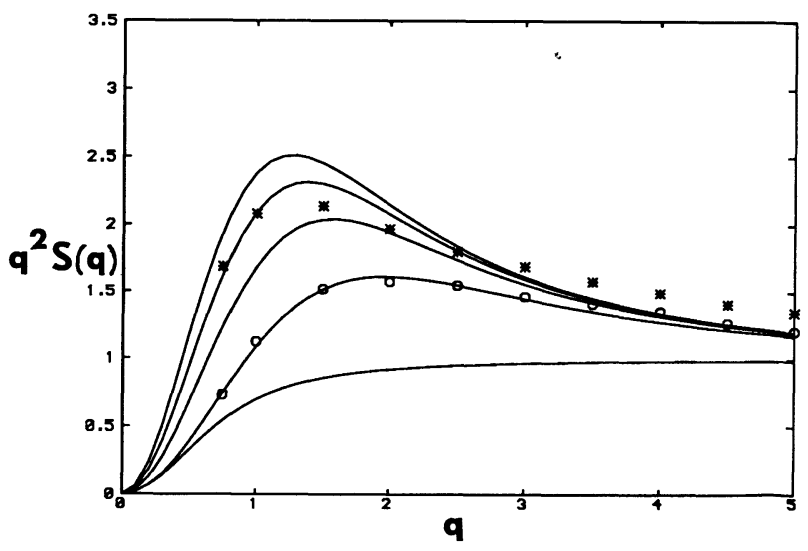

Fig. 15. - Fit of scattering data for a deswollen gel to the P.J.A. model. * Molecular weight of labelled path $M_{\mathrm{w}}=2.5 \times 10^{6} ; \bigcirc M_{\mathrm{w}}=8 \times 10^{5}$. The data has been scaled horizontally until it best fits into the series of theoretical curves. The theoretical curves are for $\lambda=4.6$ and $N_{\mathrm{s}}=24,18,12,6$ and an unstrained curve for $N_{\mathrm{s}}=6$. The data appear to correspond to $N_{\mathrm{s}}=15-16$ (long path) and $N_{\mathrm{s}}=6$ (short path). Implications of this for the apparent mesh molecular weight are discussed in the text. (Data taken from Bastide J., Herz J. and Boue F. J. Phys. (1985) [30]). 
Figure 15 shows that a good fit may be obtained by setting $\sqrt{S_{0}}=100 \AA$. The low molecular weight path $\left(M_{\mathrm{w}}=8 \times 10^{5}\right)$ matches the $N_{s}=6$ curve and the high molecular weight path $\left(M_{\mathrm{w}}=2.5 \times 10^{6}\right)$ corresponds to $N_{s}=15-16$. We note that the ratio of the two $M_{\mathrm{w}}$ values and the two $N_{s}$ values are approximately equal, which must be true if the crosslink density is the same in both cases. A more exact correspondance of these ratios cannot be expected since the labelled chains had a considerable degree of polydispersity (see Tab. I in Ref. [30]).

Although the fit appears good and the $N_{s}$ values of the two sets of data are relatively consistent between themselves it must be noted that they are inconsistent with the original estimates of Bastide et al. Measurements of the network modulus lead to an estimate of the number of crosslinks, and hence to the average subchain (or mesh) molecular weight. Mesh molecular weights in the range 20.000-50.000 were estimated, depending on the elasticity theory used. This corresponds to a range of 50-125 meshes on the $M_{\mathrm{w}}=2.5 \times 10^{6}$ chains. If $N_{s}=16$ then the mesh molecular weight is 150.000 . The average mesh end to end distance was estimated as $125 \AA$, whereas the current fitting parameter yields the value $\left(3 S_{0}\right)^{1 / 2}=170 \AA$.

A similar fitting procedure to the replica model (Fig. 16) yields a best fit at $\sqrt{S_{0}} \approx 77 \AA$. The theoretical curves are for $N_{s}=32,24,16$ and 8 . The replica curves converge relatively rapidly to the limit $q^{2} S(q)=1$, and it can be seen that no rescaling of the data will produce a fit as good as figure 15 . We therefore conclude that, if these values of $N_{s}$ are to be believed, the experimental gels are behaving more like junction affine networks than phantom networks.

Fitting of the uniaxial extension data has proved rather less successful. In reference [32] it is shown that both the perpendicular and parallel data at $\lambda=1.46$ can be matched to theoretical models, but different models and different numbers of links are used for the two directions. This is clearly unsatisfactory. At $\lambda=4.6$ the agreement with theory is even worse as is indicated by figure 1 of reference [32]. We have been unable to find any model which provides a good fit to both the perpendicular and parallel scattering data consistently, even if a reduced $N_{s}$ value is postulated.

We do not present the graphs of the fitting process for this data due to their inconclusive nature. However we do note that the $\lambda=1.46$ data is at least roughly matched by the theories and suggests a relatively large $N_{s}(\approx 50)$, and that the $\lambda=4.6$ data differs much more from the theories, and if anything, would suggest a much lower value of $N_{s}$, as is observed in the deswollen gel data, which is also equivalent to $\lambda=4.6$. We therefore conclude that simple Gaussian network treatments are not sufficient to explain these results entirely, and that problems with the theories are particularly apparent at large deformations. We suggest several possible causes for this below.

At large $q$ it is possible to obtain approximations to the scattering functions as series in $1 / q^{2}$. This has been done for all the models as it provides a useful check on the numerical integration routines. For the replica model the expansion is :

$q^{2} S(q)=1+\frac{2}{q^{2}}\left(1-\lambda^{2}-\frac{1}{N_{s}}\right)+\mathrm{O}\left(\frac{1}{q^{4}}\right)$

for $q$ in the parallel direction, and the $\lambda^{2}$ is replaced by $1 / \lambda$ for $q$ in the perpendicular direction. Hence in the parallel direction the coefficient of $1 / q^{2}$ is large and negative, whereas in the perpendicular direction it is small and positive. The perpendicular curve should therefore converge to the limit more rapidly than the parallel curve. This effect occurs in all the models, as is seen by comparing figures 13 and 14 . The experimental data is anomalous not only in that it converges very slowly at large $q$, but also in that it appears to converge more rapidly in the parallel than the perpendicular direction. The scattered intensity at high $q$ is thus higher than predicted in both directions. It is difficult to think of any effect that would explain this. The apparent shape of the curves is very dependent on the limit value of $q^{2} S(q)$ for large $q$ estimated in the experiment. We note that this normalization of the data is rather difficult to do accurately since the data never really reach the limit in the accessible $q$ range.

We have evaluated the iso-intensity contours for the P.J.A. and the replica models with $N_{s}=50$ and $\lambda=4.6$. Figure 17 shows that the replica contours are very rounded and that the P.J.A. contours are

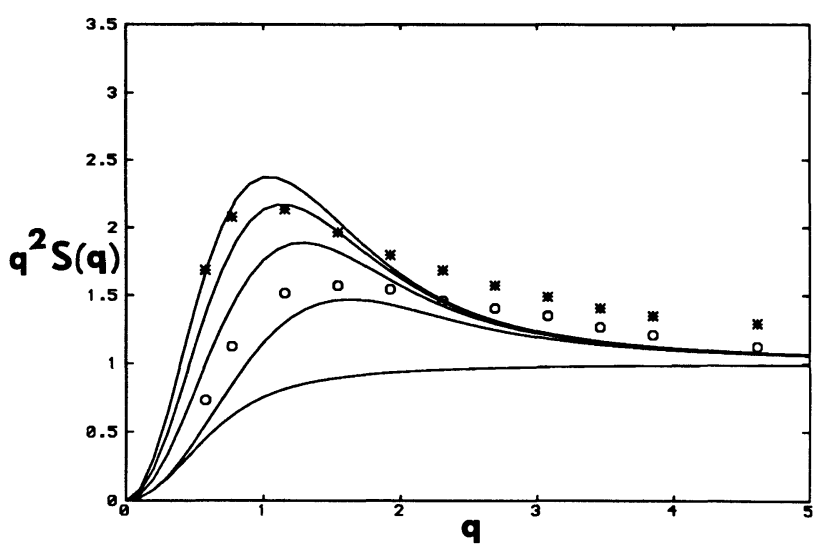

Fig. 16. - Best fit of the same data to the replica model $(C=1)$. The scaling factor used is different (see text). The theoretical curves are for $\lambda=4.6$ and $N_{\mathrm{s}}=32,24$, 16,8 and an unstrained curve for $N_{\mathrm{s}}=8$. The data do not fit so well into this series of curves. 


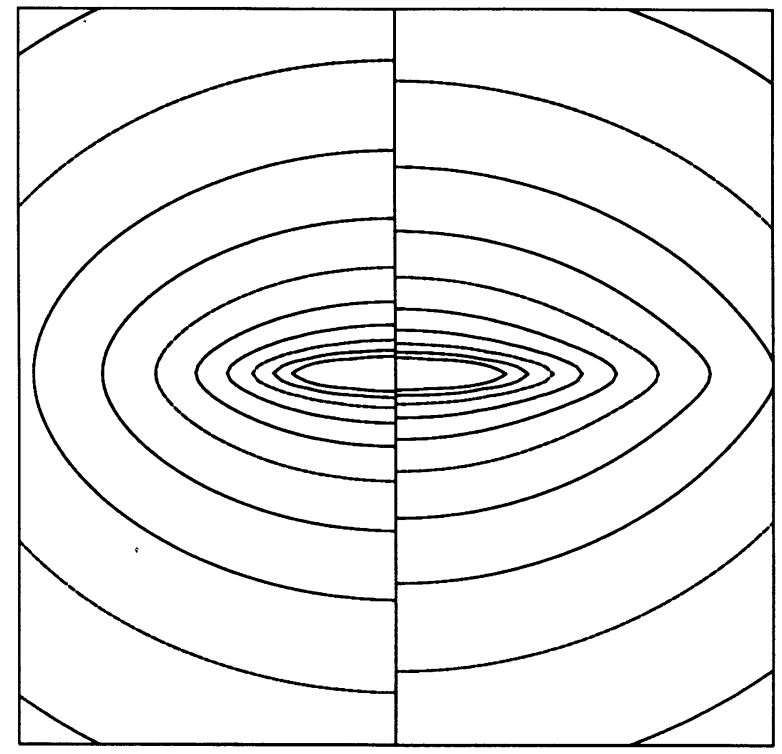

Fig. 17. - Iso-intensity contours for a uniaxially extended network with $\lambda=4.6$ and $N_{\mathrm{s}}=50$. The direction of strain is vertical. Left - replica model $(C=1)$, Right - P.J.A. The inner contour is $10 \%$ of the central intensity and each subsequent contour is reduced in intensity by a factor of 1.6. The range of $q$ is $\left|q_{\text {perp }}\right| \leqslant 5$ and $\left|q_{\text {para }}\right| \leqslant 5$. The angular junction affine contours compare more favourably with the lozenge-like experimental contours (Fig. 18).

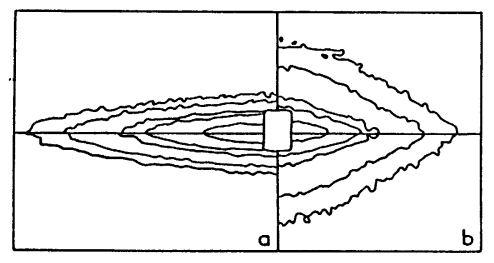

Fig. 18. - Iso-intensity contours for uniaxially extended polystyrene network at $\lambda=4.6$. Left - short relaxation time, Right - long relaxation time. Only the right hand side is intended for comparison to figure 17. (Reproduced from Bastide J. and Boue F. Physica (1986) [32]).

rather more angular. The contours obtained in experiment [32], [33] (see Fig. 18) show the surprising feature of being lozenge-like in shape at large $\lambda$. The left hand side of figure 18 shows scattering from the sample when quenched below its glass transition temperature only a short time ( 30 secs) after applying the strain. The right hand side shows scattering after a long relaxation time (30 mins). Relaxation effects are beyond the scope of this paper. The phantom network results should apply more accurately to the fully relaxed sample, and therefore only the right hand side of figure 18 is intended for comparison to figure 17. Whilst the P.J.A. contours are not exactly lozenges they are much closer to the observed shape than the rounded replica contours. Thus the iso-intensity contours agree qualitatively with the deswollen gel data that the networks are behaving more like a junction affine network than a fully fluctuating phantom network.

There are several points concerning the derivation of the scattering functions which should be borne in mind. In the P.J.A. model the number of subchains $N_{s}$ is fixed and each subchain has a range of lengths, therefore the total chain length will vary. In reality the total length of chain is fixed and $N_{s}$ varies. The problem evidently does not arise in the monodisperse models, and also not in the replica model, since the length is fixed. The P.P.N. model is a compromise between the two situations, and is slightly unsatisfactory in this respect. Thus the models differ in the way they treat the finite length of chain. The most obvious effect of this is seen at small $q$. The replica function at $q=0$ gives $S_{\text {rep }}=N_{s} / 4$. Other models differ from this by 1 part in $N_{s}$. The discrepancy is essentially an end effect. It has the same status as the unstrained dangling ends of the chain which we have already chosen to neglect. It is thus unimportant for large $N_{s}$, but may become important at small $N_{s}$ values $\leqslant 10$. In the experiments, however, the original chains were far from being exactly monodisperse, and the two effects are likely to cancel out to a large extent.

It is somewhat difficult to assess whether an exact treatment of the P.P.N. case would differ more from the replica model than does the present approximation. Figure 19 sheds some light on this matter. Curve a shows the contribution from scattering centres on the same subchain only (equivalent to the

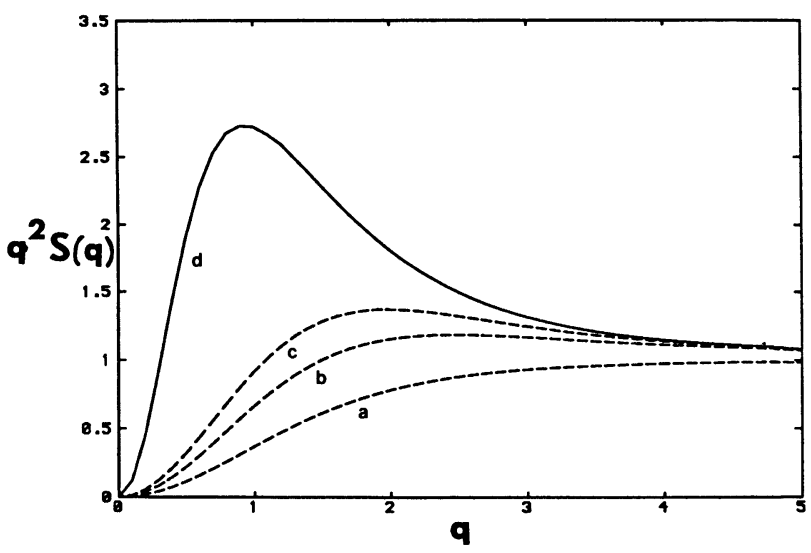

Fig. 19. - The contribution of the different terms in the P.P.N. model to the total scattering. a) scattering centres on the same subchain only; b) scattering centers on the same and neighbouring subchains ; c) scattering centers on the same, neighbouring, and next-neighbour subchains. See figure 12 ; d) final approximation to total scattering. For high $q$ the total scattering consists almost entirely of the three short distance terms. At small $q$ the long distance terms are important. 
end-linked case). Curve $b$ shows the contributions from the same and neighbouring subchains, and curve $\mathrm{c}$ includes the next neighbours in addition. These cases are shown as circuit diagrams in figure 12. In the notation of section 9 curve $c$ has the scattering function

$$
\begin{aligned}
S(\mathbf{q})=\frac{1}{4 N_{s} S_{0}^{2}}\left(N_{s}\right. & G_{\text {self }}(\mathbf{q})+ \\
& +2\left(N_{s}-1\right) G_{n=0}(\mathbf{q})+ \\
& \left.+2\left(N_{s}-2\right) G_{n=1}(\mathbf{q})\right) .
\end{aligned}
$$

Curve $d$ is the final approximation for the total scattering from the P.P.N. model. It can be seen that for $q>3$ the total scattering is represented almost entirely by these three short distance terms. However for the peak at $q \approx 1$ the majority of the scattering comes from the long distance terms for which only the approximation of (9.18) is available. The peak height is thus rather uncertain, although in this region it is bounded closely below by the lower limit curve, and it is also bounded above by the P.J.A. The true P.P.N. curve does not therefore have the slow convergence at large $q$ seen in the junction affine models, and if the peak region is to differ substantially from the approximation then it must be towards a higher, sharper peak shape, rather than a lower, broader one. Hence an exact P.P.N. calculation will not resolve the problems discussed above.

The order of the curves in figures 13 and 14 is slightly different from the reverse order which might be expected : the P.P.N. is above the replica curve in both directions. In fact at $q>5$ the P.P.N. curve in the parallel direction crosses below the replica, and indeed the $1 / q^{2}$ expansions show that at sufficiently high $q$ the order in the two figures must be strictly reversed.

The current work clearly shows that the introduction of polydispersity into the calculation of the scattering function has a marked effect. Although the P.J.A. model has some success in matching the deswollen gel data, and in predicting the angular shape of the iso-intensity contours, it is clear that the introduction of polydispersity alone is not sufficient to explain all the anomalous features of the data. Some treatment which goes beyond the idea of phantom Gaussian chains is required, since this will then not be constrained by the limiting curves calculated above.

Vilgis and Boue [28] have considered various possibilities. The theory of Flory and Erman [29] considers the major effect of topological entanglements in the network to be a restriction of the degree of fluctuation of the junction points. The form factor was calculated in accordance with this theory for an end linked network only in reference [28]. Since the model is intermediate between the fully fluctuating phantom network and the junction affine network the scattering function will lie between these bounds, and is therefore not expected to provide a significantly better fit to the data. Ball et al. [34] have modelled the effect of entanglements by «slip links ». They find that the entanglements have the largest effect at small deformations. The material behaves as though the effective number of crosslinks decreases when it is stretched. This does indeed appear to be the case from the data of reference [32]. Vilgis and Boue have calculated the scattering function for the slip link model, but again only for an end-linked network. It would therefore be of interest to extend this treatment to a labelled chain with many crosslinks.

Bastide, Herz and Boue [30] are of the opinion that the number of network defects such as pendant chains etc. is small in the gels which they use for experiment. However, computer simulations of the radiative crosslinking process by Sly and Eichinger [35] suggest that the presence of pendant chains, the formation of short wasted loops, and the scission of chains are all important factors. The scattering of a network which contains a large fraction of unstrained chains due to this type of defect would obviously be expected to be much closer to the unstrained network scattering. The consequent reduction in perpendicular scattering might be expected to be larger in long distance, small $q$ terms, and this is the region where the current theories predict too great a scattered intensity. This is therefore another possible avenue for further investigation.

We wish to stress the importance of the high $q$ region of the data $(q=3-5)$. Here the scattering predicted by any one model is essentially independent of $N_{s}$ (see Figs. 15, 16), and yet in this same region the models do differ substantially between themselves. Thus accurate data in this region would enable a distinction to be made between the models without needing to consider the value of $N_{s}$, which is not a very well known parameter. It is unfortunate that the signal to noise ratio in the experiment in this region is quite high, as is acknowledged in reference [30].

\section{Conclusions.}

We have shown that it is possible to use the analogy between resistor networks and systems of Gaussian chains to calculate the behaviour of polydisperse polymer networks. The modulus and the stress optical coefficient of a network are found to be formally independent of the distribution of chain lengths within the network. When a bimodal network is strained the chains are found to deform in such a way that the short chains are much less extended than the long ones relative to their initial root mean square length, and yet are much more extended than 
the long ones relative to their maximum length. The consequence of this is that the orientation of the chains, as measured by the Legendre polynomials $P_{2}$ and $P_{4}$, is much greater for the short chains than the long ones. The difference in response is quite large even for relatively small length ratios and should be easily experimentally observable.

The scattering function for polydisperse networks (in which the crosslinks are randomly positioned along a long labelled chain) is found to differ substantially from that for monodisperse networks (in which the crosslinks are regularly spaced along the chain). It is possible to fit the data for deswollen polystyrene gels quite well to the predictions of the Polydisperse Junction Affine model, if we assume a substantially reduced crosslink density from that originally estimated. Consideration of the shape of the iso-intensity contours for uniaxially extended gels also implies that these materials are behaving more like a junction affine network than a fully fluctuating phantom network. However there remain substantial differences between the measured intensity in the uniaxially extended systems and that predicted by any of the models considered. It has been shown by the calculation of limits on the form of the scattering function that these differences are unlikely to be explained by any treatment which does not go beyond the idea of phantom Gaussian chains.

\section{Appendix A.}

We wish to show that $\left\langle S^{\text {eff }} / S\right\rangle=2 / \phi$ for all $S$ distributions. From the circuit diagrams in section 2 we have

$$
\begin{aligned}
J & \equiv\left\langle\frac{S^{\text {eff }}}{S}\right\rangle_{\text {all subchains }} \\
& =\left\langle\frac{X+X^{\prime}}{S+X+X^{\prime}}\right\rangle=2\left\langle\frac{X^{\prime}}{S+X+X^{\prime}}\right\rangle
\end{aligned}
$$

and so

$$
\begin{aligned}
1-J / 2 & =\left\langle\frac{S+X}{S+X+X^{\prime}}\right\rangle=\left\langle\left(1+\frac{X^{\prime}}{S+X}\right)^{-1}\right\rangle \\
= & \int_{0}^{\infty} \mathrm{d} t\left\langle\exp \left(-t\left(1+\frac{X^{\prime}}{S+X^{\prime}}\right)\right)\right\rangle .
\end{aligned}
$$

Now let $u=t X^{\prime}$

$$
\begin{aligned}
1-J / 2=\int_{0}^{\infty} \mathrm{d} u & \left\langle\frac{1}{X^{\prime}} \exp \left(-u / X^{\prime}\right)\right\rangle \times \\
& \times\langle\exp (-u /(S+X))\rangle .
\end{aligned}
$$

But

$$
\frac{1}{X}=\sum_{i} \frac{1}{S_{i}+X_{i}}
$$

Therefore

$$
\langle\exp (-u / X)\rangle=\langle\exp (-u /(S+X))\rangle^{\alpha}
$$

since all the $S_{i}$ and $X_{i}$ are independent. Hence,

$$
\begin{aligned}
1-J / 2 & =\int_{0}^{\infty} \mathrm{d} u\left\langle\frac{1}{X} \exp (-u / X)\right\rangle\langle\exp (-u / X)\rangle^{1 / \alpha} \\
& =\int_{0}^{\infty} \mathrm{d} u\left\{-\frac{\mathrm{d}}{\mathrm{d} u}\langle\exp (-u / X)\rangle^{1+1 / \alpha} \frac{1}{1+1 / \alpha}\right\}=\frac{\alpha}{\alpha+1}=\frac{\phi-1}{\phi}
\end{aligned}
$$

and $J=2 / \phi$.

\section{Appendix B.}

We wish to solve (2.9) in the case where $f(S)$ has a narrow spread about its mean $S_{0}$.
Let $S=S_{0}+s$ and $X=X_{0}+x$

$$
X=\left(\sum_{i=1}^{\alpha} \frac{1}{S_{i}+X_{i}}\right)^{-1} \quad \text { from (2.8) }
$$

$$
\begin{aligned}
X_{0}+x=\left\{\sum_{i=1}^{\alpha} \frac{1}{\left(S_{0}+X_{0}\right)\left(1+\frac{s_{i}+x_{i}}{S_{0}+X_{0}}\right)}\right\}^{-1} \\
=\left(S_{0}+X_{0}\right)\left\{\sum_{i} 1-\frac{s_{i}+x_{i}}{S_{0}+X_{0}}+\left(\frac{s_{1}+x_{i}}{S_{0}+X_{0}}\right)^{2} \ldots\right\}^{-1} \\
=\frac{\left(S_{0}+X_{0}\right)}{\alpha}\left\{1+\frac{1}{\alpha} \sum_{i} \frac{s_{i}+x_{i}}{S_{0}+X_{0}}-\frac{1}{\alpha} \sum_{i}\left(\frac{s_{i}+x_{i}}{S_{0}+X_{0}}\right)^{2}+\frac{1}{\alpha^{2}}\left(\sum_{i} \frac{s_{i}+x_{i}}{S_{0}+X_{0}}\right)^{2}\right\} .
\end{aligned}
$$


Thus

$$
x=\frac{1}{\alpha^{2}} \sum_{i}\left(x_{i}+s_{i}\right)+\mathrm{O}\left(s^{2}\right) .
$$

Squaring this equation and taking expectation values gives (using the independence of all the variables $s_{i}$ and $x_{i}$ ),

$$
\text { so } \quad \begin{aligned}
\left\langle x^{2}\right\rangle & =\frac{1}{\alpha^{3}}\left(\left\langle x^{2}\right\rangle+\left\langle s^{2}\right\rangle\right) \\
\left\langle x^{2}\right\rangle & =\frac{1}{\alpha^{3}-1}\left\langle s^{2}\right\rangle .
\end{aligned}
$$

The self-consistency relation can be written approximately as :

$$
\begin{array}{rl}
g(X)=\iint \prod_{i} \mathrm{~d} s_{i} \mathrm{~d} x_{i} & f\left(s_{i}\right) g\left(x_{i}\right) \times \\
\times \delta\left(x-\frac{1}{\alpha} \sum_{i} x_{i}+s_{i}\right)
\end{array}
$$

where for convenience the same symbols $g$ and $f$ are used for the probability distributions with respect to the new variables. This can be solved by taking Fourier transforms.

$$
\begin{aligned}
& F(k)=\int_{-\infty}^{\infty} f(S) \mathrm{e}^{-i k s} \mathrm{~d} S=\left\langle\mathrm{e}^{-i k s}\right\rangle \\
& G(k)=\int_{-\infty}^{\infty} g(X) \mathrm{e}^{-i k x} \mathrm{~d} X=\left\langle\mathrm{e}^{-i k x}\right\rangle
\end{aligned}
$$

from (B.2)

$$
\begin{aligned}
\left\langle\mathrm{e}^{-i k x}\right\rangle & =\left\langle\exp \left(-\frac{i k}{\alpha^{2}} \sum_{i}\left(x_{i}+s_{i}\right)\right)\right\rangle \\
& =\left\langle\mathrm{e}^{-i k s / \alpha^{2}}\right\rangle^{\alpha}\left\langle\mathrm{e}^{-i k x / \alpha^{2}}\right\rangle^{\alpha} \\
G(k) & =F\left(k / \alpha^{2}\right)^{\alpha} G\left(k / \alpha^{2}\right)^{\alpha} .
\end{aligned}
$$

We illustrate the method using the narrow bimodal distribution of (2.20) :

$$
\begin{aligned}
f(s) & =p_{1} \delta\left(s-s_{1}\right)+p_{2} \delta\left(s-s_{2}\right) \\
& =0.5 \delta(s-0.025)+0.5 \delta(s+0.025)
\end{aligned}
$$

with $\phi=3$, (B.5) becomes :

$$
\begin{aligned}
G(k) & =F\left(\frac{k}{4}\right)^{2} G\left(\frac{k}{4}\right)^{2} \\
& =F\left(\frac{k}{4}\right)^{2} F\left(\frac{k}{16}\right)^{4} G\left(\frac{k}{16}\right)^{4} \\
& \equiv F^{*}(k) G\left(\frac{k}{16}\right)^{4} .
\end{aligned}
$$

The function $F^{*}(k)$ can easily be obtained from $f(s)$, and we may use an approximation for $G$ which is valid for the small argument $k / 16$ :

$$
\begin{aligned}
G(k) & \approx \exp \left(-k^{2}\left\langle x^{2}\right\rangle / 2\right) \\
G\left(\frac{k}{16}\right) & \approx \exp \left(-\frac{1}{2} \frac{k^{2}}{256}\left\langle x^{2}\right\rangle\right) \\
& \approx \exp \left(-\frac{1}{2} \frac{k^{2}}{256} \frac{\left\langle s^{2}\right\rangle}{7}\right)
\end{aligned}
$$

hence

$$
\begin{aligned}
g(x) \approx \frac{1}{2 \pi} & \int_{-\infty}^{\infty} \mathrm{d} k F^{*}(k) \times \\
& \times \exp \left(-\frac{2 k^{2}}{256} \frac{\left\langle s^{2}\right\rangle}{7}\right) \exp (i k x)
\end{aligned}
$$

To get back to $g(X)$ we need to shift the function along by $X_{0}$. An estimate of $X_{0}$ is obtained by averaging equation (B.1) over all the $s_{i}$ and $x_{i}$ distributions.

$$
\begin{array}{r}
X_{0}=\frac{\left(S_{0}+X_{0}\right)}{\alpha}\left\{1-\frac{(\alpha-1)}{\alpha} \frac{\left(\left\langle x^{2}\right\rangle+\left\langle s^{2}\right\rangle\right)}{\left(S_{0}+X_{0}\right)^{2}}\right\}+ \\
+\mathrm{O}\left(\left\langle s^{3}\right\rangle\right) .
\end{array}
$$

The zero order approximation is

$$
X_{0}=\left(S_{0}+X_{0}\right) / \alpha=X_{\text {mono }}
$$

otherwise, rearranging, we obtain :

$$
\begin{array}{r}
X_{0}=\frac{S_{0}}{\alpha-1}\left\{1-\frac{\alpha^{3}}{\alpha^{3}-1} \frac{\left\langle s^{2}\right\rangle}{\left(S_{0}+X_{0}\right)^{2}}\right\}+ \\
+\mathrm{O}\left(\left\langle s^{3}\right\rangle\right)
\end{array}
$$

and it is sufficient to put the $X_{0}$ on the right of the equation equal to $X_{\text {mono }}$ or $X_{M}$. Similarly we may expand the equation :

$$
\frac{1}{X_{M}}=\alpha\left\langle\frac{1}{X_{M}+S_{0}+s}\right\rangle
$$

to obtain

$$
\begin{array}{r}
X_{\mathrm{M}}=\frac{S_{0}}{\alpha-1}\left\{1-\frac{\alpha}{\alpha-1} \frac{\left\langle s^{2}\right\rangle}{\left(S_{0}+X_{0}\right)^{2}}\right\}+ \\
+\mathrm{O}\left(\left\langle s^{3}\right\rangle\right) .
\end{array}
$$

Hence $X_{\mathrm{M}}$ is always less than $X_{0}$.

\section{Acknowledgments.}

PGH is grateful for a CASE award from the Agricultural and Food Research Council and Unilever Research, and wishes to thank F. Boue for his correspondance and L. Baxandall for his helpful suggestions. RCB wishes to acknowledge a stimulating seminar from Dr. R. S. Stein. We are indebted to the referee for his careful criticism of section 10 . 


\section{References}

[1] Flory, P. J., Proc. R. Soc. A 351 (1976) 351.

[2] James, H. M. and Guth, E., J. Chem. Phys. 11 (1943) 455.

[3] James, H. M., J. Chem. Phys. 15 (1947) 651.

[4] James, H. M. and Guth, E., J. Chem. Phys. 15 (1947) 669.

[5] Mark, J. E., Adv. Polym. Sci. 44 (1982) 1.

[6] Deam, R. T. and Edwards, S. F., Philos. Trans. $R$. Soc. A 280 (1976) 317.

[7] Eichinger, B. E., Macromolecules 5 (1972) 496.

[8] Graessley, W. W., Macromolecules 8 (1975) 186 and 865 .

[9] BALl, R. C. and EdWARdS, S. F., Macromolecules 13 (1980) 748.

[10] Eichinger, B. E., Macromolecules 13 (1980) 1.

[11] De Gennes, P. G., Scaling Concepts in Polymer Physics (Cornell Univ. Press) 1979, p. 141.

[12] ThORPE, M. F., Excitations in Disordered Systems NATO ASI Series B $\mathbf{7 8}$ Ed. M. F., Thorpe (Plenum Press, New York) 1982.

[13] Stinchcombe, R. B., J. Phys. C 7 (1974) 179.

[14] Mark, J. E. and Curro, J. G., J. Chem. Phys. 79 (1983) 5705.

[15] Curro, J. G. and Mark, J. E., J. Chem. Phys. 80 (1984) 4521.

[16] Andrady, A. L., Llorente, M. A. and Mark, J. E., J. Chem. Phys. 72 (1980) 2282.

[17] Andrady, A. L., Llorente, M. A. and Mark, J. E., J. Chem. Phys. 73 (1980) 1439.
[18] KunN, W. and Grun, F., Kolloid Z. 101 (1942) 248.

[19] WARD, I. M., Structure and Properties of Oriented Polymers (Applied Science Pubs, London) 1975.

[20] STEIN, R. S., Seminar discussion.

[21] Treloar, L. R. G., The Physics of Rubber Elasticity (Clarendon Press, Oxford) 3rd ed., 1975.

[22] Roe, R. J. and Krigbaum, W. R., J. Appl. Phys. 35 (1964) 2215.

[23] Erman, B. and Flory, P. J., Macromolecules 16 (1983) 1601 and 1607.

[24] Mitchell, G. R., Br. Polym. J. 17 (1985) 111.

[25] Pearson, D. S., Macromolecules 10 (1977) 696.

[26] Ullman, R., Macromolecules 15 (1982) 1395 and corrective note 16 (1983) 1400.

[27] Warner, M. and Edwards, S. F., J. Phys. A. 11 (1978) 1649.

[28] Vilgis, T. and Boue, F., Polymer 27 (1986) 1154.

[29] Flory, P. J. and ERman, B., Macromolecules 15 (1982) 801.

[30] Bastide, J., Herz, J. and Boue, F., J. Phys. France 46 (1985) 1967.

[31] Ball, R. C., Thesis, Univ. Cambridge (1980).

[32] Bastide, J. and Boue, F., Physica 104A (1986) 251.

[33] Boue, F., Bastide, J., Buzier, M., Colette, C., LAPP, A. and HERZ, J., preprint (1987).

[34] BALl, R. C., DoI, M., EDWARDS, S. F. and WARner, M., Polymer 22 (1981) 1010.

[35] SHY, L. Y. and EICHINGER, B. E., Macromolecules 19 (1986) 2787. 